\title{
Coupling reduced-order blood flow and cardiac models through energy-consistent strategies: modeling and discretization
}

\author{
Jessica Manganotti ${ }^{1,2}$, Federica Caforio ${ }^{1,2,3}$, François Kimmig ${ }^{1,2} \mathbb{B}$, Philippe Moireau ${ }^{1,2^{*}}$ (D) and \\ Sebastien Imperiale ${ }^{1,2}$ (D)
}

${ }^{*}$ Correspondence:

philippe.moireau@inria.fr

${ }^{2}$ LMS, Ecole Polytechnique, CNRS, Institut Polytechnique de Paris, 91128 Palaiseau, France Full list of author information is available at the end of the article

\begin{abstract}
In this work we provide a novel energy-consistent formulation for the classical 1D formulation of blood flow in an arterial segment. The resulting reformulation is shown to be suitable for the coupling with a lumped (OD) model of the heart that incorporates a reduced formulation of the actin-myosin interaction. The coupling being consistent with energy balances, we provide a complete heart-circulation model compatible with thermodynamics hence stable numerically and informative physiologically. These latter two properties are verified by numerical experiments.
\end{abstract}

Keywords: Reduced-order models, Cardiovascular modelling, Energy-preserving time-scheme, Shallow-water models, Heart models, Dicrotic notch, Ageing

\section{Introduction}

The importance of reduced-order (RO) models in clinical applications has been extensively assessed in the last years. In particular, $\mathrm{RO}$ models are nowadays very widespread in the scientific literature [1-5] concerning cardiovascular applications for patient-specific model predictions. Lumped-parameter zero-dimensional (OD) models-typically Windkessel models [6-8] — can provide a general view on the global response, e.g. in pressure and flow, of the whole cardiovascular system or a portion of it $[4,9]$. Hence, RO models can be used as simplified limit conditions for a more detailed system, e.g. a full threedimensional (3D) heart model [10-13] and in practice they are often used to represent the circulation upstream and/or downstream of the domain of a higher-order model and to define the relationship between pressure and flow at its boundaries [14-16].

RO models used in cardiovascular applications are one-dimensional (1D) models of the blood circulation. The 1D formulation accounts for the effects due to pulse wave transmission and thus enables to predict important markers such as pressure wave velocity. These models have been validated against in vitro $[17,18]$ and in vivo $[19,20]$ measurements and have proven to provide useful insights for the understanding of cardiovascular physiology and pathology. Further, 1D models may be preferred over 0D models when local vascular changes or distributed properties (e.g. tapering, branching, stenoses) are under study, and

c The Author(s) 2021. This article is licensed under a Creative Commons Attribution 4.0 International License, which permits use, sharing, adaptation, distribution and reproduction in any medium or format, as long as you give appropriate credit to the original author(s) and the source, provide a link to the Creative Commons licence, and indicate if changes were made. The images or other third party material in this article are included in the article's Creative Commons licence, unless indicated otherwise in a credit line to the material. If material is not included in the article's Creative Commons licence and your intended use is not permitted by statutory regulation or exceeds the permitted use, you will need to obtain permission directly from the copyright holder. To view a copy of this licence, visit http://creativecommons.org/licenses/by/4.0/. 
when the impact of physiological and disrupted wave transmission on the circulation (f.e. the origin and clinical relevance of the dicrotic notch, as in [21]) is investigated. Therefore, 1D models are ideal to simulate blood flow in a single arterial segment $[14,20,22,23]$ or a more structured tree of arteries [16-18,24]. In addition, 1D models may also be used to take into account other components (e.g. coronary circulation) [19] and be employed to simulate the global human circulation [25-28] (in combination with lumped-parameter models for the heart dynamics [29], pulmonary circulation and microvascular beds).

In addition, $\mathrm{RO}$ models can also be employed to model the heart dynamics. The simplest approach to model the heart dynamics is based on the use of time-varying elastance heart dynamics models [30]. More accurate RO approaches were also developed, see for instance $[31,32]$. In the 0D model proposed in [32], the geometry of the left ventricle is considered to be a thick sphere and the dependence on space of the heart motion is only related to the radius of the sphere. In [31], the mechanics of ventricular interaction is based on the assumption of a simplified ventricular composite geometry. In more detail, ventricular geometry is approximated by three thick-walled spherical segments encapsulating the LV and RV cavities.

Due to their reduced computational cost, RO models are well suited for real-time monitoring when coupled with data assimilation strategies [33-35]. Furthermore, due to the reduced number of parameters used to describe the reduced dynamics (compared to full 3D models for instance), they are more adequate for the stable solution of inverse problems (IP). However, IP strategies and data assimilation strategies may fail if the forward problem lacks of appropriate stability properties. Typically, the forward model must provide a stable solution, especially when it is solved with various sets of parameters and (noisy) feedback terms. In this regard, energy-preserving schemes are ideal to discretize forward problems, since they are stable with respect to a variation of parameters and they ensure a reliable control on the behavior of the solution [36]. Moreover, energy balance and exchanges turn out to be important physiological markers that should be well approximated and be easily accessed, thus motivating once more the use of RO models that preserve the energy balance intrinsic to the considered modeled phenomena.

Several difficulties must be overcome when deriving energy-preserving RO models and their associated discretization. First at the continuous level, RO models do not necessarily come under a form that is obviously compliant with energy principles (appropriate energy balance may be lost during the model reduction). Hence, such RO models must be adapted or transformed. Then, it is also not obvious to construct a numerical scheme that preserves the continuous energy balance, since advanced RO models are often highly non-linear.

In this work, we extend the previous work of [29] by introducing a coupling strategy involving the heart model of [32] and an arterial segment. On the one hand, we provide a novel energy-consistent formulation for the classical 1D formulation of blood flow in an arterial segment. Of note, although the application envisaged blood circulation, this energy-consistent mathematical framework and the formulation proposed are prone to extension to other non-linear hyperbolic 1D problems, like shallow water equations. The resulting reformulation is shown to be suitable for the coupling with the lumped (OD) model of the heart initially proposed in the work of [32]. On the other hand, the novelty concerning the cardiac model is that we are able to prove the stability both in the continuous and discrete reduced order formulations, hence guaranteeing the consistency with the energy relation described by [36] for a 3D heart model. The coupling being consistent 
with energy balances, we finally provide a complete heart-circulation model compatible with thermodynamics hence stable numerically and informative physiologically.

The paper is structured as follows: first, in "Partial differential equations of the reduced models" section we present the equations that describe, respectively, the 1D blood flow model, the OD heart model and the valve dynamics, and we report their energy relation for all the compartments. Then, in "An energy-compliant formulation for the blood flow model" section we detail the changes of variables that are performed in the blood flow model to obtain an energy-compliant formulation and we illustrate its non trivial numerical aspects. The key aspects of the discretization are presented in "Discretization" section. Finally, in "Simulations and results" section we show the results of the numerical simulations and we draw the conclusions and perspectives.

\section{Partial differential equations of the reduced models}

\section{One-dimensional blood flow model}

Blood propagation in the aorta

Momentum and continuity equations in their one-dimensional formulation are widely used to model the arterial tree, or a portion of it, and to study pressure and blood flow [19]. The standard formulation that describes blood flow propagation in a vessel is derived from the Navier-Stokes equation by an asymptotic analysis procedure [2,37]. For all time $t>0$ we look for a blood flow $Q(t, s)$ and a cross-section $A(t, s)$, along the axis $s$ of the vessel, that are solution of

$$
\left\{\begin{array}{l}
\partial_{t} A+\partial_{s} Q=0, \\
\partial_{t} Q+\alpha_{\mathrm{vp}} \partial_{s} \frac{Q^{2}}{A}+\frac{A}{\rho} \partial_{s} P(A)+K_{r} \frac{Q}{A}=0, \\
P(A)=P_{\mathrm{ext}}+\psi_{e}(A)+\psi_{v}(A),
\end{array}\right.
$$

where $\alpha_{\mathrm{vp}}$ is a coefficient related to the a-priori assumption on the velocity profile in the vessel, $P(A)$ is the pressure, $P_{\text {ext }}$ the exterior pressure, $\rho$ the density of blood (considered as a constant value) and $K_{r}$ the friction parameter. In this work we assume that the velocity profile is flat, therefore

$$
\alpha_{\mathrm{vp}}=1 \text {. }
$$

The first equation of System (1) represents a reduced form of the continuity equation, whereas the second one corresponds to the momentum conservation and the last one is a relation that accounts for the vessel wall displacement. In particular, it links the change in pressure to the wall deformation and deformation rate, hence the change in the cross-section $A$. It reads:

$$
\psi_{e}(A)=\beta \frac{\sqrt{A}-\sqrt{A_{0}}}{A_{0}} \quad \text { and } \quad \psi_{\nu}(A)=\frac{\Gamma}{A_{0} \sqrt{A}} \partial_{t} A,
$$

where $\beta=\left(4 \sqrt{\pi} E h_{0}\right) / 3$, with $E$ the Young modulus of the vessel and $h_{0}$ its thickness, $\Gamma=\left(2 \sqrt{\pi} h_{0} v\right) / 3$, with $v$ viscosity of the wall and $A_{0}$ the reference area of the crosssection of the vessel. The analysis of $\psi_{\nu}$ will be addressed in "Viscosity of the wall" section. Therefore, if not specified, in the following sections $\psi$ corresponds to $\psi_{e}$.

System (1) should be completed with the initial condition $A(0, s)=A_{0}$ and $Q(0, s)=0$ as well as boundary conditions that are the subject of the forthcoming sections. When $\Gamma$ and $K_{r}$ are considered equal to zero, System (1) is composed by non-linear hyperbolic equations and discontinuities may appear in time, e.g. shocks, even when smooth data are 
considered. However, the presence of viscosity smoothens the solution and it is reasonable to assume that its derivative with respect to $s$ exists.

It is possible to show that, for smooth solutions, an energy relation holds. Surprisingly, such relation has not yet been used in the literature to deduce numerical models for the propagation of blood flow, although it is well known that energy-consistent methods provide great benefits in terms of stability, which is a key aspect in the context of multiphysics couplings.

Considering the continuous problem, if we take into account a vessel of length $L$, then we can define the energy related to $(A, Q)-$ or to $(A, u)$ where $u$ is the blood velocity since $Q=A u-$ as

$$
\mathcal{E}_{\mathrm{ar}}(t)=\int_{0}^{L} e(t, s) \mathrm{d} s
$$

with

$$
e=\frac{\rho}{2} A u^{2}+\Psi(A) \text { and } \Psi(A(t, s))=\int_{A_{0}}^{A(t, s)} \psi_{e}(a) \mathrm{d} a \geq 0 .
$$

Moreover, we define the instantaneous loss term as

$$
\mathcal{D}_{\mathrm{ar}}(t)=\rho K_{r} \int_{0}^{L} u^{2} \mathrm{~d} s+\frac{\Gamma}{A_{0}} \int_{0}^{L} \frac{\left|\partial_{s}(A u)\right|^{2}}{\sqrt{A}} \mathrm{~d} s \geq 0 .
$$

Then, a straightforward extension of [37, Lemma 2.2] can be deduced.

Lemma 1 Any smooth solution of System (1) satisfies the conservation property

$$
\frac{\mathrm{d}}{\mathrm{d} t} \mathcal{E}_{a r}+\mathcal{D}_{a r}+\left.Q\left(P_{t o t}-P_{\text {ext }}\right)\right|_{0} ^{L}=0,
$$

with $P_{\text {tot }}$ defined as

$$
P_{t o t}=P(A)+\frac{\rho}{2} u^{2} .
$$

\section{Outflow and inflow conditions}

The energy balance (5) shows that the energy defined in (3) is a decreasing function of time if there is no blood flow imposed at the inlet and the outlet. But of course, System (1) should be completed with more realistic boundary conditions, typically relating the input flux (output flux, respectively) or the pressure described in (6) at the inlet (outlet, respectively) with the flux or pressure in other systems. In our case, a simple three-element Windkessel [38] is employed at the outlet, hence we introduce a new unknown, a pressure $P_{c}$, that satisfies

$$
C_{c} \frac{\mathrm{d}}{\mathrm{d} t} P_{c}+\frac{P_{c}}{R_{\text {per }}}=Q(L), \quad\left(P_{\text {tot }}-P_{\text {ext }}\right)-P_{c}=R_{c} Q(L),
$$

where $C_{c}, R_{\text {per }}$ and $R_{c}$ are positive parameters that correspond to a conductance and resistances in the Windkessel terminology. At the inlet, we have

$$
P_{\text {ar }}=P_{\text {tot }}(0)-P_{\text {ext }}, \quad Q_{\text {ar }}=Q(0),
$$

where $P_{\mathrm{ar}}$ and $Q_{\mathrm{ar}}$ stand for the arterial pressure and the arterial flux at the inlet of the aorta, respectively. One can then easily deduce from Lemma 1 an energy balance for the coupled Eqs. (1) and (7).

Theorem 2 Any smooth solution of Systems $(1,7)$ satisfies the conservation property

$$
\frac{\mathrm{d}}{\mathrm{d} t}\left(\mathcal{E}_{a r}+\mathcal{E}_{w}\right)+\mathcal{D}_{a r}+\mathcal{D}_{w}=P_{a r} Q_{a r}
$$


where

$$
\mathcal{E}_{w}=\frac{C_{c}}{2} P_{c}^{2}, \quad \mathcal{D}_{w}=\frac{P_{c}^{2}}{R_{p e r}}+R_{c} Q(L)^{2} .
$$

In the next two subsections a non-linear reduced-model of the heart and cardiac valves is derived as an efficient parametrized generator of an inlet flow. This reduced model will then be adequately coupled to the one-dimensional model proposed in this work in order to preserve the energy balance.

\section{Heart model}

In this section, we describe the chosen reduced heart model proposed in [32]. This model benefits from an appealing mathematical structure while producing accurate pressurevolume loops. It integrates in a system of ODEs a microscopical Huxley-like model of actinmyosin binding with a macroscopical cavity deformation formulation. The dimension reduction relies on a spherical hypothesis and a shell asymptotic derivation. Here, we will prove that an energy balance exists for the reduced model as it is the case for the threedimensional formulation that is derived in [36]. This will allow us to derive a complete energy balance property when the $0 \mathrm{D}$ heart model is coupled to the 1D blood flow.

\section{Cardiac mechanics}

Following [32], we can derive by an asymptotic procedure a system of ODEs describing a lumped cardiac mechanical model that is geometrically represented by a thick sphere of radius $R$ and thickness $d$-see Fig. 2. The unknown displacement field is reduced to a radial lumped quantity $y$, that is a unique scalar variable such that the deformed radius of the sphere $R$, the thickness in the deformed configuration $d$ and the volume of the deformed cavity $V$ [39], shown in Fig. 2, are given by

$$
R(y)=R_{0}+y, \quad d(y)=d_{0}\left(1+\frac{y}{R_{0}}\right)^{-2} \text { and } V(y)=\frac{4}{3} \pi\left(R(y)-\frac{d(y)}{2}\right)^{3},
$$

where $R_{0}$ and $d_{0}$ are the radius and the thickness of the sphere in the reference configuration, respectively. The system of ODEs also involves variables accounting for the modeling of heart contraction through the active deformation $e_{c}$ and the active stress $\tau_{c}$, which are linked to the global deformation with the rheology pictured in Fig. 2 following the recent formulation proposed in Kimmig et al. [40]. The system is loaded with the ventricular pressure $P_{v}$. The dynamics reads

$$
\left\{\begin{array}{l}
\rho_{0}\left|\Omega_{0}\right| \ddot{y}+\frac{\left|\Omega_{0}\right|}{R_{0}} k_{s}\left(\frac{y}{R_{0}}-e_{c}\right)+\frac{\partial \mathcal{W}_{p}}{\partial y}(y)+\mathcal{W}_{v}(y, \dot{y})=P_{v} \frac{\partial V(y)}{\partial y}, \\
\mu \dot{e}_{c}-k_{s}\left(\frac{y}{R_{0}}-e_{c}\right)=-\tau_{c},
\end{array}\right.
$$

where $\rho_{0}$ is the density in the reference configuration, $\mu$ a viscosity parameter, $k_{s}$ a stiffness parameter accounting for passive components of the myosin filament-typically the passive stiffness of the filaments themselves plus the $Z$-disks and $\left|\Omega_{0}\right|$ is the volume of the myocardium in the reference configuration, which is given by

$$
\left|\Omega_{0}\right|=\frac{4}{3} \pi\left[\left(R_{0}+\frac{d_{0}}{2}\right)^{3}-\left(R_{0}-\frac{d_{0}}{2}\right)^{3}\right] .
$$

Moreover $\mathcal{W}_{p}(y)$ and $\mathcal{W}_{v}(y, \dot{y})$ are directly inferred from the passive potential and the viscous pseudo-potential of the connective tissue matrix [32]. They are smooth functions 
that satisfy in particular

$$
\mathcal{W}_{p}(y) \geq 0 \quad \text { and } \quad \mathcal{W}_{v}(y, \dot{y}) \dot{y} \geq 0 .
$$

Choosing an isotropic transverse exponential law $[32,41]$ leads to

$$
\begin{aligned}
& \mathcal{W}_{p}(y)=\left|\Omega_{0}\right|\left[C_{0} e^{C_{1}\left[2\left(1+\frac{y}{R_{0}}\right)^{2}+\left(1+\frac{y}{R_{0}}\right)^{-4}-3\right]^{2}}+C_{2} e^{\left.C_{3}\left[\left(1+\frac{y}{R_{0}}\right)^{2}-1\right]^{2}\right]},\right. \\
& \mathcal{W}_{v}(y, \dot{y})=2 \eta\left|\Omega_{0}\right|\left(1+\frac{y}{R_{0}}\right)^{2}\left[1+2\left(1+\frac{y}{R_{0}}\right)^{-12}\right] \dot{y},
\end{aligned}
$$

where $C_{0}$ and $C_{2}$ are some parameters that describe the stiffness of the material and $C_{1}$ and $C_{3}$ are non-dimensional parameters, while $\eta$ is the viscosity.

Assuming for now that the active stress $\tau_{c}$ is imposed, the dynamics described in System (10) gives a reduced heart dynamical systems of 2-state variables $\left(y, e_{c}\right)$ for which we can derive the heart energy balance. We first introduce the energy

$$
\mathcal{E}_{\mathrm{hr}}=\frac{\rho_{0}\left|\Omega_{0}\right|}{2} \dot{y}^{2}+\mathcal{W}_{p}+\frac{\left|\Omega_{0}\right| k_{s}}{2}\left(\frac{y}{R_{0}}-e_{c}\right)^{2},
$$

that is a combination of the kinetic energy $\rho_{0}\left|\Omega_{0}\right| \frac{\dot{y}^{2}}{2}$, the hyperelastic energy $\mathcal{W}_{p}$ and the elastic energy stored in the series element. Then, we introduce the dissipation term

$$
\mathcal{D}_{\mathrm{hr}}=\mathcal{W}_{v}(y, \dot{y}) \dot{y}+\mu\left|\Omega_{0}\right|\left(\dot{e}_{c}\right)^{2}
$$

and state the following energy relation result.

Theorem 3 Any smooth solution of System (10) satisfies the energy balance

$$
\frac{\mathrm{d}}{\mathrm{d} t} \mathcal{E}_{h r}+\mathcal{D}_{h r}=P_{v} \frac{\mathrm{d}}{\mathrm{d} t}(V(y))-\left|\Omega_{0}\right| \tau_{c} \dot{e}_{c},
$$

where, at the right-hand side, we have the coupling term with the circulation and the microscopic active stress input.

\section{Microscopic actin-myosin binding model}

The chosen model of active contraction balances our need of reasonable complexity with physiological characteristics regarding pressure-volume loops $[36,40,42]$. In more detail, the microscopic active stress $\tau_{c}$ is computed from the first two moments of a Huxley-like formulation of the actin-myosin binding phenomenon. Introducing the active stiffness variable $k_{c}$, we have the following system

$$
\left\{\begin{array}{l}
\dot{\tau}_{c}=-\left(|v|+\alpha\left|\dot{e}_{c}\right|\right) \tau_{c}+n_{0}\left(e_{c}\right) \sigma_{0}|v|_{+}+k_{c} \dot{e}_{c} \\
\dot{k}_{c}=-\left(|v|+\alpha\left|\dot{e}_{c}\right|\right) k_{c}+n_{0}\left(e_{c}\right) k_{0}|v|_{+}
\end{array}\right.
$$

where we use the symbol $|\cdot|_{+}$to denote the positive part. The parameter $k_{0}$ denotes the maximum active stiffness parameter and $\sigma_{0}$ the corresponding maximum active stress, $\alpha$ is a time constant, $n_{0}\left(e_{c}\right)$ is a function with values in $[0,1]$ accounting for the Frank-Starling mechanism, and $v(t)=v\left(\left[\mathrm{Ca}^{2+}\right](t)\right)$ is a function triggering the contraction, typically when $\left[\mathrm{Ca}^{2+}\right]>c_{\text {th }}$, with $c_{\text {th }}$ a given threshold, see Fig. 1 .

The state variables $\left(\tau_{c}, k_{c}\right)$ of System (14) model the active contraction triggered by the input signal $v$. For the energy balance, we introduce the energy stored in our homogenized model of actin-myosin bridges and a dissipative term [36]

$$
\mathcal{E}_{c}=\frac{\left|\Omega_{0}\right|}{2} \frac{\tau_{c}^{2}}{k_{c}}, \quad \mathcal{D}_{c}=\left(|v|+\alpha\left|\dot{e}_{c}\right|\right) \mathcal{E}_{c}
$$


(a)

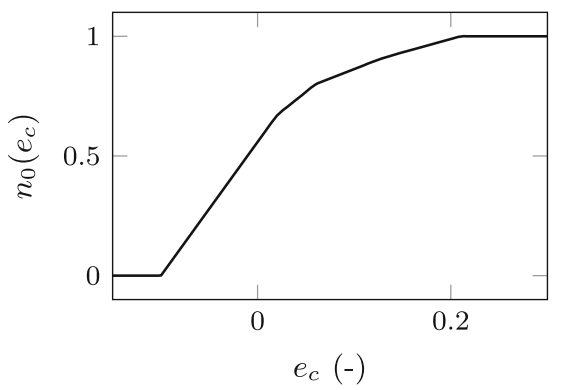

(b)

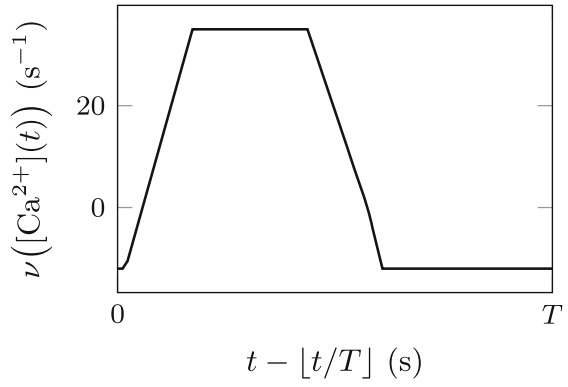

Fig. 1 a Definition of the function $n_{0}$. $\mathbf{b}$ Time evolution of contraction triggering function $v$. We denote here by $T$ the duration of a heartbeat

Theorem 4 Any smooth solution of System (14) satisfies the energy balance

$$
\frac{\mathrm{d}}{\mathrm{d} t} \mathcal{E}_{c}+\mathcal{D}_{c}=\left|\Omega_{0}\right| \tau_{c} \dot{e}_{c}+\frac{\left|\Omega_{0}\right| n_{0}\left(e_{c}\right) \tau_{c}}{k_{c}}\left(\sigma_{0}-\frac{\tau_{c} k_{0}}{2 k_{c}}\right)|v|_{+} .
$$

Combining (13) and System (15), we finally obtain the complete energy balance for the heart model

$$
\frac{\mathrm{d}}{\mathrm{d} t}\left[\mathcal{E}_{\mathrm{hr}}+\mathcal{E}_{c}\right]+\left[\mathcal{D}_{\mathrm{hr}}+\mathcal{D}_{c}\right]=P_{v} \frac{\mathrm{d}}{\mathrm{d} t}(V(y))+\frac{\left|\Omega_{0}\right| n_{0}\left(e_{c}\right) \tau_{c}}{k_{c}}\left(\sigma_{0}-\frac{\tau_{c} k_{0}}{2 k_{c}}\right)|v|_{+},
$$

where, from the point of view of the heart model, $v$ is an input signal and $P_{v}$ an external loading.

Remark 5 The energy relation in System (15) is deduced by introducing the variable $\lambda_{c}=\tau_{c} / \sqrt{k_{c}}$ [36]. Then, as an intermediate step it can be shown that $\lambda_{c}$ satisfies the following ODE:

$$
\dot{\lambda}_{c}=-\frac{\dot{k}_{c}}{2 k_{c}} \lambda_{c}+\frac{\dot{\tau}_{c}}{\sqrt{k_{c}}}=-\left(|v|+\alpha\left|\dot{e}_{c}\right|\right) \frac{\lambda_{c}}{2}+\frac{n_{0}\left(e_{c}\right)}{\sqrt{k_{c}}}\left(\sigma_{0}-\frac{k_{0} \lambda_{c}}{2 \sqrt{k_{c}}}\right)|v|_{+}+\sqrt{k_{c}} \dot{e}_{c} .
$$

The energy relation is finally obtained by multiplying (16) by $\left|\Omega_{0}\right| \lambda_{c}$.

\section{Cardiac valve models and energy relation for the complete system}

The inlet and outlet of the ventricular model are represented, respectively, by the atrioventricular and the aortic valve, as shown in Fig. 2. To represent the fact that they may be open or closed, valves are modeled as diodes. Therefore, using Kirchhoff's circuit laws, we get

$$
\left\{\begin{array}{l}
C_{\mathrm{mi}} \dot{P}_{v}-Q_{v}+\frac{\left|P_{v}-P_{\mathrm{ar}}\right|_{+}}{K_{\mathrm{ar}}}+\frac{\left|P_{v}-P_{\mathrm{at}}\right|_{+}}{K_{\mathrm{iso}}}-\frac{\left|P_{\mathrm{at}}-P_{v}\right|_{+}}{K_{\mathrm{at}}}=0, \\
C_{\mathrm{ar}} \dot{P}_{\mathrm{ar}}-\frac{\left|P_{v}-P_{\mathrm{ar}}\right|_{+}}{K_{\mathrm{ar}}}+Q_{\mathrm{ar}}=0,
\end{array}\right.
$$

where the first equation is associated with the atrioventricular valve, whereas the second one relates to the aortic valve. In more detail, $P_{v}, P_{\text {at }}$ and $P_{\text {ar }}$ represent the pressure in the ventricle, in the atria and at the inlet of the aorta, respectively, whereas $Q_{v}$ and $Q_{\mathrm{ar}}$ correspond to the ejected blood flow throughout the ventricle and at the inlet of the aorta, respectively. Finally, $K_{\mathrm{ar}}, K_{\mathrm{iso}}$ and $K_{\mathrm{at}}$ represent the resistances of the valves [43], as depicted in Fig. 2. 


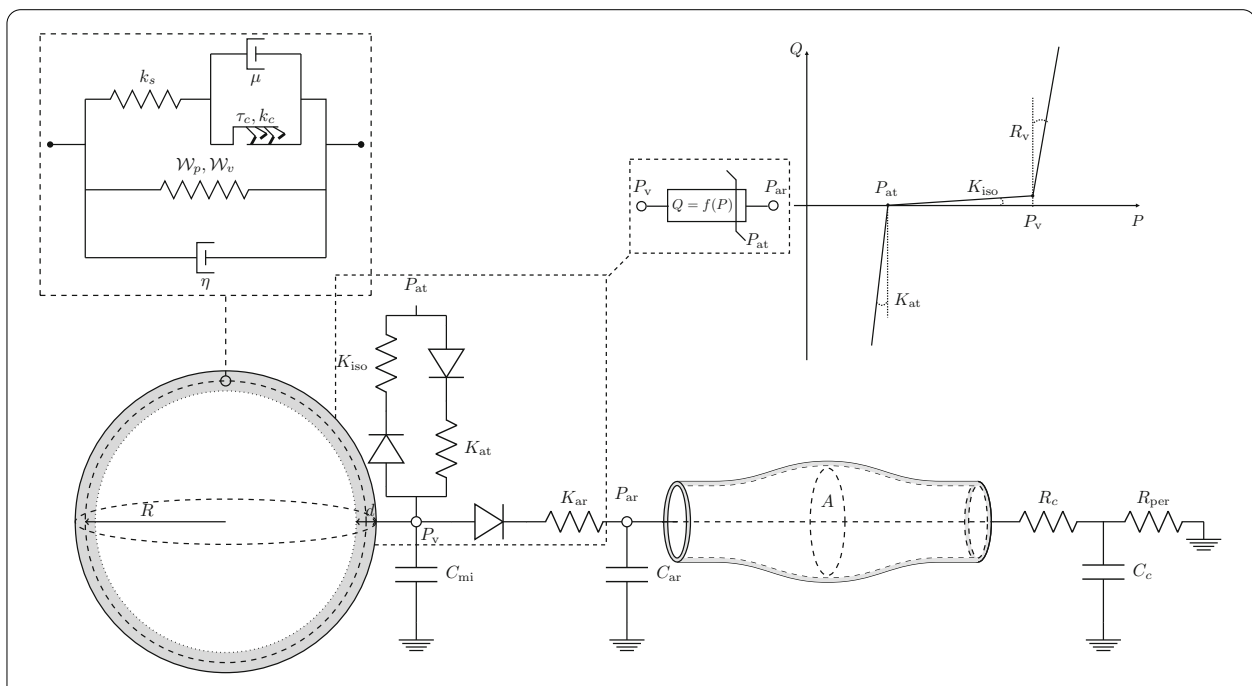

Fig. 2 Complete scheme of the coupled system including the heart, the cardiac valve and the arterial segment. Note that in the upper right plot we have $R_{v}:=K_{a r} K_{\text {iso }} /\left(K_{a r}+K_{\text {iso }}\right)$. This model will later be referred to as the fully coupled model

It is worth mentioning, for what follows, that $Q_{v}$ corresponds to the volume variation of the ventricle, so one can write

$$
-\frac{\mathrm{d}}{\mathrm{d} t}(V(y))=Q_{v}
$$

Now, if we multiply the first equation of System (17) by $P_{v}$, we multiply the second equation by $P_{\text {ar }}$, we sum them and we define

$$
\mathcal{E}_{v}=\frac{C_{\mathrm{mi}}}{2} P_{v}^{2}+\frac{C_{\mathrm{ar}}}{2} P_{\mathrm{ar}}^{2}, \quad \mathcal{D}_{v}=\frac{\left|P_{v}-P_{\mathrm{ar}}\right|_{+}^{2}}{K_{\mathrm{ar}}}+\frac{\left|P_{v}-P_{\mathrm{at}}\right|_{+}^{2}}{K_{\text {iso }}}+\frac{\left|P_{\mathrm{at}}-P_{v}\right|_{+}^{2}}{K_{\mathrm{at}}},
$$

we can obtain an energy relation for the cardiac valve formulation.

Theorem 6 Any smooth solution of System (17) satisfies the conservation property

$$
\frac{\mathrm{d}}{\mathrm{d} t} \mathcal{E}_{v}+\mathcal{D}_{v}=\frac{\left|P_{a t}-P_{v}\right|_{+} P_{a t}}{K_{a t}}-\frac{\left|P_{v}-P_{a t}\right|_{+} P_{a t}}{K_{i s o}}-P_{v} \frac{\mathrm{d}}{\mathrm{d} t}(V(y))-P_{a r} Q_{a r}
$$

where we have, at the right-hand side, the input pressure term and the coupling term that take into account both the cardiac and the arterial contribution.

Finally, using Theorems 2 to 6 , we are able to retrieve the global energy relation that takes into account the contribution of the heart (including the microscopic modeling of the actin-myosin binding), the valves and the arterial segment.

Corollary 7 Any smooth solution of Systems (1, 7, 10, 14, 17) satisfies the conservation property

$$
\begin{aligned}
& \frac{\mathrm{d}}{\mathrm{d} t}\left(\mathcal{E}_{h r}+\mathcal{E}_{c}+\mathcal{E}_{v}+\mathcal{E}_{a r}+\mathcal{E}_{w}\right)+\mathcal{D}_{h r}+\mathcal{D}_{c}+\mathcal{D}_{v}+\mathcal{D}_{a r}+\mathcal{D}_{w} \\
& \quad=\frac{\left|\Omega_{0}\right| n_{0}\left(e_{c}\right) \tau_{c}}{k_{c}}\left(\sigma_{0}-\frac{\tau_{c} k_{0}}{2 k_{c}}\right)|v|_{+}+\frac{\left|P_{a t}-P_{v}\right|_{+} P_{a t}}{K_{a t}}-\frac{\left|P_{v}-P_{a t}\right|_{+} P_{a t}}{K_{i s o}}
\end{aligned}
$$




\section{An energy-compliant formulation for the blood flow model}

In order to obtain an energy-preserving scheme for the blood flow model, which satisfies a discrete counterpart of Theorem 1, we will introduce three variational formulations. The first one corresponds to the standard formulation that one obtains directly from System (1). This formulation has $A$ and $u$ as principal unknowns, where $A$ is the crosssection of the aorta defined as $A=Q / u$ and $u$ is the blood velocity. Then, we introduce a second formulation that uses as a primary unknown the radius of the aorta $R=\sqrt{A} / \sqrt{\pi}$ and $u$. This formulation is straightforwardly deduced from the first one and is a convenient intermediate step, since it introduces several simplifications. From these intermediate changes we deduce the last formulation that is written in the unknowns

$$
v:=R u \quad \text { and } \quad \Phi:=\varphi(R),
$$

where $\varphi(R)$ is a smooth bijective function from $\mathbb{R}^{+}$to $I \subset \mathbb{R}$ that we define later. This change of variables has the main advantage to provide an "energy-compliant" discretization, as it will be shown in the next chapter. As the reader will see, the energy is a quadratic functional of the new variables $(\Phi, v)$.

\section{Variational formulation in $(A, u)$}

As a first step, we substitute $Q$ with $A u$ in (1) and assume that $\Gamma=0$, hence $\psi_{\nu}=0$. After some algebraic manipulations we obtain that (1) is equivalent to the system

$$
\left\{\begin{array}{l}
\partial_{t} A+\partial_{s}(A u)=0 \\
\left(\frac{1}{2}\left(\partial_{t} A\right) u+A \partial_{t} u\right)+\left(\frac{1}{2} u^{2} \partial_{s} A+\frac{3}{2} A u \partial_{s} u\right)+\frac{A}{\rho} \partial_{s} P(A)+K_{r} u=0 .
\end{array}\right.
$$

Note that we have rewritten System (1) in a specific form adapted to the derivation of the energy balance (in fact System (21) is obtained following the proof of Lemma 1 provided in [37]). Indeed, multiplying the second equation of System (21) by $u$, one can see that

$$
\begin{aligned}
& \left(\frac{1}{2}\left(\partial_{t} A\right) u+A \partial_{t} u\right) u=\frac{1}{2} \partial_{t}\left(A u^{2}\right), \\
& \text { and }\left(\frac{1}{2} u^{2} \partial_{s} A+\frac{3}{2} A u \partial_{s} u\right) u=\frac{1}{2} \partial_{s}\left(A u^{3}\right) .
\end{aligned}
$$

These two equalities are, in fact, essential to prove the energy relation of Lemma 1. The objective is now to derive a weak formulation of System (21). Concerning the first equation, we multiply it by a space-dependent test function $\tilde{\Phi}$ and we integrate in space, obtaining

$$
\left(\partial_{t} A, \tilde{\Phi}\right)+\left(\partial_{s}(A u), \tilde{\Phi}\right)=0,
$$

where $(\cdot, \cdot)$ is the $L^{2}$-scalar product in $(0, L)$. We now focus on the second equation of System (21) and we repeat the procedure performed above, multiplying each term by a space-dependent test function $\tilde{v}$. After some manipulations, we obtain

$$
\begin{aligned}
& \left(\frac{\rho}{2}\left(\partial_{t} A\right) u+\rho A \partial_{t} u, \tilde{v}\right)+a(u ; \tilde{v}, A) \\
& \quad-\left(\partial_{s}(A \tilde{v}), \psi(A)\right)+\rho K_{r}(u, \tilde{v})=g(\tilde{v} ; A, u),
\end{aligned}
$$

where $a(u ; \cdot, \cdot)$ is bilinear in its two last arguments but non-linear in $u$ and is given by

$$
a(u ; A, \tilde{v}):=\int_{0}^{L} \rho\left(\frac{1}{2} u^{2} \partial_{s} A+\frac{3}{2} A u \partial_{s} u\right) \tilde{v} \mathrm{~d} s-\left.\frac{\rho}{2} A u^{2} \tilde{v}\right|_{0} ^{L},
$$


and the non-linear functional $g$ is defined as

$$
g(A, u ; \tilde{v}):=-\left.\left(\frac{\rho}{2} A u^{2} \tilde{v}+A \tilde{v} \psi(A)\right)\right|_{0} ^{L}=-\left.A \tilde{v}\left(P_{\text {tot }}-P_{\text {ext }}\right)\right|_{0} ^{L} .
$$

Observe that, by construction, $g$ is linear in $\tilde{v}$ and includes only boundary terms. Up to this point, the weak formulation of the problem described in System (21) is

$$
\left\{\begin{array}{l}
\left(\partial_{t} A, \tilde{\Phi}\right)+\left(\partial_{s}(A u), \tilde{\Phi}\right)=0, \\
\left(\frac{\rho}{2}\left(\partial_{t} A\right) u+\rho A \partial_{t} u, \tilde{v}\right)+a(u ; A, \tilde{v})-\left(\partial_{s}(A \tilde{v}), \psi(A)\right)+\rho K_{r}(u, \tilde{v})=g(A, u ; \tilde{v}) .
\end{array}\right.
$$

Finally, observe that if we substitute

$$
\tilde{\Phi}=\psi(A), \quad \tilde{v}=u,
$$

in System (27), we can easily retrieve the energy relation presented in Lemma 1. Indeed, thanks to (22), one can see that

$$
a(u ; A, u)=0 .
$$

Moreover, we recover the energy relation

$$
\frac{\mathrm{d}}{\mathrm{d} t} \mathcal{E}_{a r}(t)+\mathcal{D}_{a r}(t)=g(A, u ; u)
$$

with

$$
\frac{\mathrm{d}}{\mathrm{d} t} \mathcal{E}_{a r}(t)=\left(\frac{\rho}{2}\left(\partial_{t} A\right) u+\rho A \partial_{t} u, u\right)+\left(\partial_{t} A, \psi(A)\right) \quad \text { and } \quad \mathcal{D}_{a r}(t)=\rho K_{r}(u, u) .
$$

Here $\mathcal{E}_{a r}(t)$ is the total energy of the $1 \mathrm{D}$ model and $\mathcal{D}_{a r}(t)$ represents the dissipative term with $K_{r} \geq 0$.

\section{An intermediate formulation in $(R, u)$}

In order to obtain a formulation that leads to the achievement of the energy preservation at a discrete level, we construct an intermediate form of System (21). This formulation is obtained by replacing $A$ with $\pi R^{2}$, where $R$ represents the radius of the lumen. The unknowns become $u$ and $R$. The first equation of System (21) is now described as

$$
2 \pi\left(R \partial_{t} R, \tilde{\Phi}\right)+\pi\left(\partial_{s}\left(R^{2} u\right), \tilde{\Phi}\right)=0 .
$$

Then, the first term in (24) can be rewritten, substituting $\tilde{v} \leftarrow \tilde{v} / R$, as

$$
\left(\frac{\rho}{2}\left(\partial_{t} A\right) u+\rho A \partial_{t} u, \frac{\tilde{v}}{R}\right)=\pi \rho\left(u \partial_{t} R+R \partial_{t} u, \tilde{v}\right)=\pi \rho\left(\partial_{t}(R u), \tilde{v}\right) .
$$

Moreover, one can see that

$$
a\left(u ; A, \frac{\tilde{v}}{R}\right)=\pi \rho \int_{0}^{L}\left(\frac{(R u)^{2}}{R^{2}} \partial_{s} R+\frac{3}{2}(R u) \partial_{s} \frac{R u}{R}\right) \tilde{v} \mathrm{~d} s-\left.\pi \frac{\rho}{2}(R u)^{2} \frac{\tilde{v}}{R}\right|_{0} ^{L},
$$

and

$$
\left(\partial_{s}\left(A \frac{\tilde{v}}{R}\right), \psi(A)\right)=\pi\left(\partial_{s}(R \tilde{v}), \psi\left(\pi R^{2}\right)\right) .
$$

Note that the substitution $\tilde{v} \leftarrow \tilde{v} / R$ does not lead to any issue, since we consider solutions with $R>0$ at any time and position. Finally, collecting the four expressions above, one can obtain a formulation with $R$ and $u$ as primary unknowns. It reads

$$
\left\{\begin{array}{l}
2 \pi\left(R \partial_{t} R, \tilde{\Phi}\right)+\pi\left(\partial_{s}\left(R^{2} u\right), \tilde{\Phi}\right)=0 \\
\pi \rho\left(\partial_{t}(R u), \tilde{v}\right)+a\left(u ; \pi R^{2}, \frac{\tilde{v}}{R}\right) \\
\quad-\pi\left(\partial_{s}(R \tilde{v}), \psi\left(\pi R^{2}\right)\right)+\rho K_{r}\left(u, \frac{\tilde{v}}{R}\right)=g\left(\pi R^{2}, u ; \frac{\tilde{v}}{R}\right) .
\end{array}\right.
$$


It is worth noticing that the product $R u$ appears "almost" naturally and it is therefore tempting to define $v:=R u$ as a new variable. It becomes even more obvious that this choice is suitable by looking at the energy density, defined as

$$
e=\frac{\rho}{2} A u^{2}+\Psi(A)=\frac{\pi \rho}{2}(R u)^{2}+\Psi\left(\pi R^{2}\right) .
$$

This is precisely what motivates the introduction of the next formulation. Moreover, it is worth mentioning that now

$$
\psi\left(\pi R^{2}\right)=\beta \frac{\sqrt{\pi} R-\sqrt{A_{0}}}{A_{0}},
$$

so $\psi\left(\pi R^{2}\right)$ is linear with respect to the unknown $R$ and we will see in the next sections that $\Psi\left(\pi R^{2}\right)$ is a third-order polynomial and this will simplify its analysis.

Remark 8 The change of variable $A=\pi R^{2}$ is still meaningful even if the $1 \mathrm{D}$ hemodynamic model does not assume a perfect circle for the geometry of the cross-section. What matters here is that the new variable $R$ depends on the square root of $A$. Obviously, the introduction of the factor $\pi$ is natural to obtain a physical meaning for this new variable since, in practice, arterial cross-sections are almost circular.

\section{Variational formulation in $(\Phi, v)$}

A change of variables has to be made in order to demonstrate that the scheme is energypreserving after time discretization. More precisely, time discretization can easily deal with energies that involve quadratic terms of the unknowns. However, the energy density described in (33) is not a quadratic term of the unknowns $(R, u)$, but we can see that the first contribution is a quadratic term of

$$
v:=R u
$$

Therefore, we propose to use $v$ as a main unknown. A first naive choice is then to set $\varphi(R)$ equal to $\sqrt{\Psi\left(\pi R^{2}\right)}$, where $\Psi\left(\pi R^{2}\right)$ is defined as

$$
\Psi\left(\pi R^{2}\right)=\int_{A_{0}}^{A} \beta \frac{\sqrt{a}-\sqrt{A_{0}}}{A_{0}} d a=\frac{\beta}{A_{0}}\left[\frac{2}{3} \pi^{\frac{3}{2}} R^{3}-\sqrt{A}_{0} \pi R^{2}+\frac{1}{3} A_{0}^{\frac{3}{2}}\right],
$$

and set $\Phi \equiv \varphi(R)$ as the other main unknown. However, we show in "Variational formulation in $(\Phi, v)$ " section that this choice is not convenient, since $\varphi(\cdot)$ would not be a bijective function from $\mathbb{R}^{+}$to $\mathbb{R}^{+}$. Instead, we define

$$
\varphi(R):=\left\{\begin{array}{ll}
\sqrt{\Psi\left(\pi R^{2}\right)} & R \geq R_{0}, \\
-\sqrt{\Psi\left(\pi R^{2}\right)} & 0 \leq R<R_{0},
\end{array} \quad \text { with } \quad R_{0}=\frac{\sqrt{A_{0}}}{\sqrt{\pi}} .\right.
$$

Before studying in more detail the impact of the choice described in System (36) (in particular the bijectivity of the function $\varphi$ ), we formally give the variational formulation associated with the new couple of unknowns $(v, \Phi)$, where $\Phi:=\varphi(R)$. Assuming for now that $\varphi$ is bijective, we define the reciprocal function $r(\Phi):=\varphi^{-1}(\Phi)$. Then, each term of the second equation of System (32) can be modified as follows:

i. The term involving the time derivative reads

$$
\pi \rho\left(\partial_{t}(R u), \tilde{v}\right)=\pi \rho\left(\partial_{t} v, \tilde{v}\right)
$$


ii. The non-linear transport term reads

$$
\begin{aligned}
a\left(u ; \pi R^{2}, \frac{\tilde{v}}{R}\right) & =\tilde{a}\left(\frac{v}{r(\Phi)}, v, \tilde{v}\right) \\
& :=\frac{\pi \rho}{2} \int_{0}^{L}\left(2 \tilde{v} \frac{v}{r(\Phi)} \partial_{s} v+v \tilde{v} \partial_{s} \frac{v}{r(\Phi)}\right) \mathrm{d} s-\left.\frac{\pi \rho}{2} \tilde{v} v \frac{v}{r(\Phi)}\right|_{0} ^{L},
\end{aligned}
$$

where $\tilde{a}$ is now a trilinear form. Such reformulation will lead to the choice of an adapted space discretization that preserves, for all sufficiently smooth functions $v$ and $u$, the property

$$
\tilde{a}(u, v, v)=0,
$$

and in particular for $u=v / r(\Phi)$.

iii. The coupling term $\left(\partial_{S}(R \tilde{v}), \psi\left(\pi R^{2}\right)\right)=\left(\partial_{S}(r(\Phi) \tilde{v}), \psi\left(\pi r(\Phi)^{2}\right)\right)$ is not modified.

iv. The dissipation term reads

$$
\left(u, \frac{\tilde{v}}{R}\right)=\left(\frac{v}{r(\Phi)}, \frac{\tilde{v}}{r(\Phi)}\right)
$$

v. Finally, the boundary term $g$ is given by

$$
g\left(\pi R^{2}, u ; \frac{\tilde{v}}{R}\right)=\tilde{g}(r(\Phi), \tilde{v}):=-\left.\pi \tilde{v} r(\Phi)\left(P_{\text {tot }}-P_{\text {ext }}\right)\right|_{0} ^{L},
$$

where, for simplicity, we assume that $P_{\text {tot }}$ is given. Of course, if more general boundary conditions are considered, $g$ must be modified accordingly.

Using all the expressions above, we obtain the following equation (corresponding to the second equation of System (32))

$$
\pi \rho\left(\partial_{t} v, \tilde{v}\right)+\tilde{a}\left(\frac{v}{r}, v, \tilde{v}\right)-\pi\left(\partial_{s}(r \tilde{v}), \psi\left(\pi r^{2}\right)\right)+\rho K_{r}\left(\frac{v}{r}, \frac{\tilde{v}}{r}\right)=\tilde{g}(r, \tilde{v}),
$$

where, for the sake of clarity, we have written $r$ instead of $r(\Phi)$. The first term in (42) clearly shows how the introduction of $v$ simplifies the dynamic behavior of the equation and it will help at the discrete level to demonstrate the energy preservation. Now we deal with the first equation of System (32) in which we use as a test function $\tilde{\Phi} \leftarrow \xi(R) \tilde{\Phi}$, with

$$
\xi(R):=\frac{\psi\left(\pi R^{2}\right)}{\varphi(R)} .
$$

We show in "Variational formulation in $(\Phi, v)$ " section that this function is smooth and positive. We obtain

$$
2 \pi\left(\xi(R) R \partial_{t} R, \tilde{\Phi}\right)+\pi\left(\xi(R) \partial_{s}\left(R^{2} u\right), \tilde{\Phi}\right)=0 .
$$

If we focus on the first term in (44), we can observe that

$$
2 \pi \xi(R) R \partial_{t} R=\pi \frac{\psi\left(\pi R^{2}\right)}{\varphi(R)} \partial_{t} R^{2}=\frac{\partial_{t} \Psi\left(\pi R^{2}\right)}{\varphi(R)} .
$$

Now observe that, by definition, $\varphi(R)= \pm \sqrt{\Psi\left(\pi R^{2}\right)}$. Thus, the term above can be rewritten as

$$
2 \pi \xi(R) R \partial_{t} R=2 \partial_{t} \varphi(R) .
$$

Since by definition we have $\Phi=\varphi(R)$ and $R=r(\Phi)$, we can write

$$
2 \pi\left(\xi(R) R \partial_{t} R, \tilde{\Phi}\right)+\pi\left(\xi(R) \partial_{s}\left(R^{2} u\right), \tilde{\Phi}\right)=2\left(\tilde{\Phi}, \partial_{t} \Phi\right)+\pi\left(\partial_{s}(r v), \tilde{\Phi} \xi(r)\right),
$$


where again we use the convention $r \equiv r(\Phi)$. At this point, the formulation reads

$$
\left\{\begin{array}{l}
2\left(\tilde{\Phi}, \partial_{t} \Phi\right)+\pi\left(\partial_{s}(R v), \tilde{\Phi} \xi(R)\right)=0, \\
\pi \rho\left(\partial_{t} v, \tilde{v}\right)+\tilde{a}\left(\tilde{v}, v, \frac{v}{R}\right)-\pi\left(\partial_{s}(R \tilde{v}), \psi\left(\pi R^{2}\right)\right)+\rho K_{r}\left(\frac{v}{R}, \frac{\tilde{v}}{R}\right)=\tilde{g}(\tilde{v}, R), \\
R=r(\Phi) .
\end{array}\right.
$$

One can see in System (47) an apparent lack of symmetry. Indeed, one could expect the second term in the first equation to be equal to the third term in the second equation. This is true however, since we have, using (43),

$$
\left(\partial_{s}(R \tilde{v}), \psi\left(\pi R^{2}\right)\right)=\left(\partial_{s}(R \tilde{v}), \varphi(R) \xi(R)\right)=\left(\partial_{s}(R \tilde{v}), \Phi \xi(R)\right) .
$$

This observation is fundamental to obtain an energy estimate. To summarize, we have deduced from the dynamics (32) the following formulation:

for all $(\tilde{\Phi}, \tilde{v})$ sufficiently smooth find, for all $t>0,(\Phi(t), v(t))$ solution of

$$
\left\{\begin{array}{l}
2\left(\tilde{\Phi}, \partial_{t} \Phi\right)+\pi\left(\partial_{s}(R v), \tilde{\Phi} \xi(R)\right)=0 \\
\pi \rho\left(\partial_{t} v, \tilde{v}\right)+\tilde{a}\left(\tilde{v}, v, \frac{v}{R}\right)-\pi\left(\partial_{s}(R \tilde{v}), \Phi \xi(R)\right)+\rho K_{r}\left(\frac{v}{R}, \frac{\tilde{v}}{R}\right)=\tilde{g}(\tilde{v}, R), \\
R=r(\Phi)
\end{array}\right.
$$

with the following initial data

$$
\Phi(0)=\varphi(R(0))=\varphi\left(\sqrt{A}_{0} / \sqrt{\pi}\right)=0, \quad v=0 .
$$

This is what we call the energy-compliant variational formulation. At the continuous level, the energy is easily obtained by choosing $\tilde{\Phi}=\Phi$ and $\tilde{v}=v$. This simple choice of test functions to deduce the energy relation at the continuous level will help in achieving the same energy relation property at a discrete level.

Remark 9 The formulation of System (48) can be obtained for other tube laws $\psi(A)$. However, some properties should be satisfied by the function $\psi$. In particular, $\psi$ must be at least continuous and

$$
\psi^{\prime}(A)>0, \quad \psi\left(A_{0}\right)=0 .
$$

\section{Strong formulation}

For the sake of completeness, we show the strong formulation of System (48). Choosing a smooth test function with compact support in $[0, L]$, one can show, using integration by parts, that the following partial differential equations hold:

$$
\left\{\begin{array}{l}
2 \partial_{t} \Phi+\pi \xi(R) \partial_{s}(R v)=0, \\
\pi \rho \partial_{t} v+\frac{\pi \rho}{2}\left(2 \frac{v}{R} \partial_{s} v+v \partial_{s} \frac{v}{R}\right)+\rho K_{r} \frac{v}{R^{2}}+\pi R \partial_{s}(\xi(R) \Phi)=0, \\
R=r(\Phi) .
\end{array}\right.
$$

Then, choosing a smooth test function in $[0, L]$ vanishing at the boundaries in System (48) and using integration by parts for System (50), one can deduce the following boundary conditions:

$$
\frac{\rho}{2} \frac{v^{2}}{R}+R \xi(R) \Phi=-R\left(P_{\text {tot }}-P_{\text {ext }}\right), \quad s \in\{0, L\}
$$

\section{Analysis of the function $\varphi(R)$}

In this section we provide further details on the properties of the function $\varphi(R)$. The definition in System (36) is motivated by the expression of $\Psi=\Psi\left(\pi R^{2}\right)$ that is rewritten 

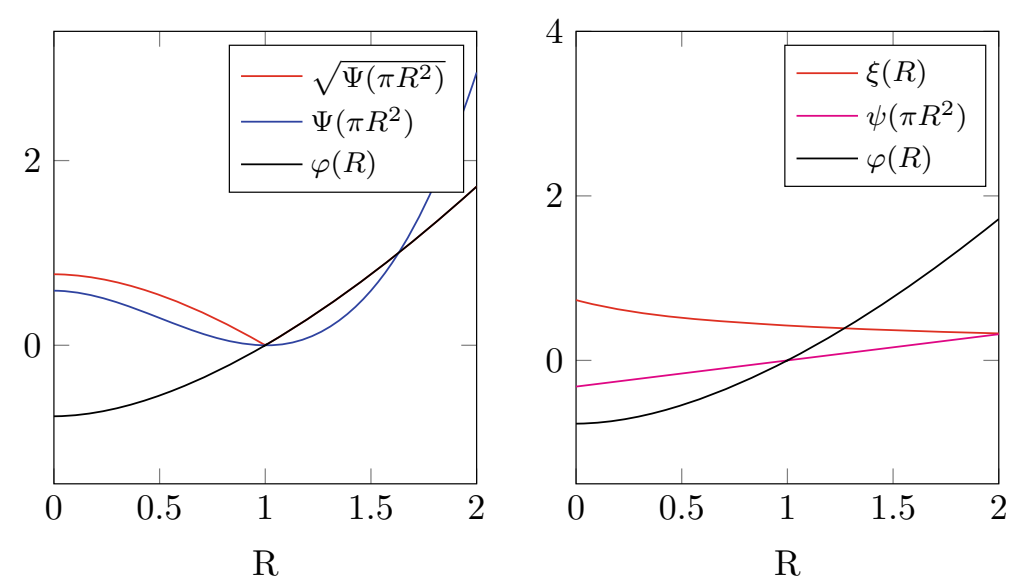

Fig. 3 Plot of $\sqrt{\Psi(R)}, \Psi(R), \varphi(R)$ and $\xi(R)$ for $R_{0}=1$ and $\beta=1$

below:

$$
\Psi\left(\pi R^{2}\right)=\frac{\beta}{A_{0}}\left[\frac{2}{3} \pi^{\frac{3}{2}} R^{3}-\sqrt{A_{0}} \pi R^{2}+\frac{1}{3} A_{0}^{\frac{3}{2}}\right]=\frac{\sqrt{\pi} \beta}{3 R_{0}^{2}}\left(R-R_{0}\right)^{2}\left(2 R+R_{0}\right),
$$

where-in this section $-R_{0}=\sqrt{A_{0}} / \sqrt{\pi}$ is the reference radius of the cross-section. The behavior of this function is shown in Fig. 3. It is straightforward to see that this function, as well as its square root, is not bijective. However, using System (36), the function $\varphi(R)$ is then given by

$$
\varphi(R)=\frac{\left(R-R_{0}\right)}{R_{0}} \sqrt{\frac{\sqrt{\pi} \beta}{3}\left(2 R+R_{0}\right)} .
$$

In Fig. 3 we can observe the comparison between $\left.\sqrt{\Psi\left(\pi R^{2}\right.}\right)$ and $\varphi(R)$. For every $R \geq R_{0}$ the two functions coincide, whereas for $R<R_{0}$ they are opposite. However, we can also see that $\varphi$ is bijective from $\mathbb{R}$ to some interval $I$ satisfying $\mathbb{R}^{+} \subset I \subset \mathbb{R}$. Moreover, it is easy to prove the following Property.

Theorem 10 Assume $R_{0}>0$, then

$$
\begin{aligned}
\varphi:[0,+\infty) & \mapsto\left[\Phi_{\text {min }},+\infty\right) \\
R & \mapsto \quad \varphi(R)
\end{aligned} \quad \text { with } \quad \Phi_{\text {min }}=-\sqrt{\frac{\beta \sqrt{\pi} R_{0}}{3}}
$$

is monotone increasing (hence bijective) and belongs to $C^{\infty}([0,+\infty))$.

\section{Analysis of the function $\xi(R)$}

We now focus on the property of the function $\xi(R)$ that is defined by $\xi(R)=\psi\left(\pi R^{2}\right) / \varphi(R)$. In particular we want to check whether the function is smooth and bounded. This is not true because $\varphi(R)$ vanishes and, as one can see in Fig. 3 and in (52), this happens at $R=R_{0}$ where-in this section $-R_{0}=\sqrt{A_{0}} / \sqrt{\pi}$ is the reference radius of the cross-section. Using Eqs. (34) and (52) one can compute

$$
\xi(R)=\frac{\beta \sqrt{\pi}}{\pi R_{0}^{2}} \frac{R-R_{0}}{\varphi(R)}=\frac{\sqrt{3 \beta}}{\pi^{\frac{3}{4}} R_{0} \sqrt{\left(2 R+R_{0}\right)}} .
$$

We see in Fig. 3 that $\xi(R)$ has no singularity, it is smooth, strictly positive and monotonically decaying. This result is summarized in the following Property. 
Theorem 11 Assume $R_{0}>0$, then

$$
\begin{aligned}
\xi:[0,+\infty) & \longrightarrow\left(0, \sqrt{\frac{3 \beta}{\pi^{\frac{3}{2}} R_{0}^{3}}}\right] \\
R & \mapsto \xi(R)
\end{aligned}
$$

is monotone decreasing, strictly positive, and belongs to $C^{\infty}([0,+\infty))$.

\section{Extension of the model in a non-physiological range}

There is an equivalence between System (27) and System (48). More precisely, we can state the following Theorem.

Theorem 12 Let $P_{\text {tot }}(t) \in C^{0}([0, T])$ be given. We have the following results:

- Let $(A, u) \in C^{1}([0, T] \times[0, L])^{2}$ be solution of System (27). If $A>0$ and if we define $R=\sqrt{A} / \sqrt{\pi}$, then $(\boldsymbol{\Phi}(R), R u) \in C^{1}([0, T] \times[0, L])^{2}$ is solution of System (48).

- Reciprocally, if $(\Phi, v)$ is solution of System (48) and if

$$
\Phi>\Phi_{\text {min }}
$$

then $\left(\pi r(\Phi)^{2}, v / r(\Phi)\right)$ is solution of System (27).

Although the bound defined in (53) is expected physiologically, after space discretization there is no guarantee that such property holds intrinsically at any time and any point. Therefore, we propose to modify System (48) for a non-physiological range, e.g. close to $R \simeq 0$, or equivalently, $\Phi \simeq \Phi_{\text {min }}$. More precisely, $r(\Phi)$ is not defined for $\Phi$ taking smaller values than $\Phi_{\min }$. To circumvent this problem we introduce, for a given $\epsilon>0$-a relaxation parameter-the function $r_{\epsilon}$, defined by

$$
r_{\epsilon}(\Phi):=\left\{\begin{array}{ll}
r(\Phi) & \Phi \geq \Phi_{\epsilon}, \\
a e^{-b \Phi} & \Phi<\Phi_{\epsilon},
\end{array} \quad \text { with } \quad \Phi_{\epsilon}=\Phi_{\text {min }}+\epsilon,\right.
$$

where $(a, b) \in \mathbb{R}^{2}$ are only defined by the constraint that $r_{\epsilon} \in C^{1}(\mathbb{R})$. In more detail, one needs to check that

$$
r\left(\Phi_{\epsilon}\right)=a e^{-b \Phi_{\epsilon}}, \quad(r)^{\prime}\left(\Phi_{\epsilon}\right)=-a b e^{-b \Phi_{\epsilon}},
$$

hence one can compute that

$$
b=-\frac{(r)^{\prime}\left(\Phi_{\epsilon}\right)}{r\left(\Phi_{\epsilon}\right)} \quad \text { and then } \quad a=e^{b \Phi_{\epsilon}} r\left(\Phi_{\epsilon}\right) .
$$

The main advantage of using $r_{\epsilon}$ instead of $r$ is that $r_{\epsilon}$ is a bijective function from $\mathbb{R}$ to $\mathbb{R}^{+} \backslash\{0\}$. Hence, at the discrete level for any value of the unknown $\Phi$ we are able to compute a corresponding aortic radius $R$. In this process we introduce-mathematically speaking-a modeling error with respect to System (27). Nevertheless, we have the following straightforward result.

Theorem 13 Let $P_{\text {tot }}(t) \in C^{0}([0, T])$ be given and $A_{\epsilon}=\pi\left[r_{\epsilon}\left(\Phi_{\epsilon}\right)\right]^{2}$. We have the following results:

- Let $(A, u) \in C^{1}([0, T] \times[0, L])^{2}$ be solution of System (27). If $A \geq A_{\epsilon}$ and if we define $R=\sqrt{A} / \sqrt{\pi}$, then $(\boldsymbol{\Phi}(R), R u) \in C^{1}([0, T] \times[0, L])^{2}$ is solution of System (48) with $r_{\epsilon}$ instead of $r$. 
- Reciprocally, if $(\Phi, v)$ is solution of System (48) with $r_{\epsilon}$ instead of $r$ and if

$$
\Phi \geq \varphi_{\epsilon},
$$

then $\left(\pi r_{\epsilon}(\Phi)^{2}, v / \varphi_{\epsilon}(\Phi)\right)$ is solution of System (27).

Note that we can choose $\epsilon$ small enough so that the range of values $A(x, t) \in\left(0, A_{\epsilon}\right)$ for which the mathematical equivalence with System (27) is not satisfied can be set as desired. In particular, considering the application to hemodynamics, this interval can be chosen so that a solution of System (27) with values $A<A_{\epsilon}$ is outside the validity of the the tube law described in (2).

\section{Viscosity of the wall}

In "One-dimensional blood flow model" section we introduced the third equation of System (1) that relates the pressure with the strain and strain rate of the wall. In particular, it takes into account the velocity of radial displacements [34] thanks to the term $\psi_{v}$ that was assumed to vanish in "Variational formulation in $(A, u)$ " section in order to derive the energy-compliant variational formulation. In this section we address the treatment of this term, $\psi_{v}$, through the change of variables introduced in "An energy-compliant formulation for the blood flow model" section. Starting from System (21), we have

$$
\frac{A}{\rho} \partial_{s} P(A)=\frac{A}{\rho} \partial_{s}\left(\psi_{e}(A)+\psi_{v}(A)\right)=\frac{A}{\rho} \partial_{s}\left(\frac{\beta}{A_{0}}\left(\sqrt{A}-\sqrt{A_{0}}\right)+\frac{\Gamma}{A_{0} \sqrt{A}} \partial_{t} A\right) .
$$

Since we have already dealt in the previous section with the first term, related to $\psi_{e}$, we focus now on the last one of the equation above, related to $\psi_{\nu}$. Starting from (56) and using the first equation of System (21), we obtain

$$
\frac{A}{\rho} \partial_{s}\left(\frac{\Gamma}{A_{0} \sqrt{A}} \partial_{t} A\right)=-\frac{A}{\rho} \partial_{s}\left(\frac{\Gamma}{A_{0} \sqrt{A}} \partial_{s} Q\right) \text {. }
$$

This motivates the introduction of the non-linear form $c(\cdot ; \cdot, \cdot)$ defined by

$$
c(R ; v, \tilde{v})=\frac{\sqrt{\pi} \Gamma}{R_{0}^{2}} \int_{0}^{L} \frac{1}{R} \partial_{s}(R \tilde{v}) \partial_{s}(R v) \mathrm{d} s .
$$

Taking into account the manipulations performed in "An intermediate formulation in $(R, u)$ " and "Variational formulation in $(\Phi, v)$ " sections, one can show that

$$
-\left(\frac{A}{\rho} \partial_{s}\left(\frac{\Gamma}{A_{0} \sqrt{A}} \partial_{s} Q\right), \rho \frac{\tilde{v}}{R}\right)=c(R ; v, \tilde{v})+\left.\pi R \psi_{v}(A) \tilde{v}\right|_{0} ^{L} .
$$

Then, it can be shown that the second equation of System (48) can be replaced by

$$
\pi \rho\left(\partial_{t} v, \tilde{v}\right)+\tilde{a}\left(\tilde{v}, v, \frac{v}{R}\right)-\pi\left(\partial_{s}(R \tilde{v}), \Phi \xi(R)\right)+c(R ; v, \tilde{v})+\rho K_{r}\left(\frac{v}{R}, \frac{\tilde{v}}{R}\right)=\tilde{g}(\tilde{v}, R) .
$$

Note that the boundary terms in (59) are indeed taken into account, since $\tilde{g}$-defined in (41) - involves the total pressure that is given by (6) and now reads

$$
P_{\text {tot }}=P_{\text {ext }}+\psi_{e}(A)+\psi_{v}(A)+\frac{\rho}{2} u^{2} .
$$

\section{Outflow conditions, inflow conditions and energy relation}

In order to complete the weak formulation of the problem given in System (48), the outflow and inflow conditions need to be specified. This is done by expanding the term $\tilde{g}$ using the coupling condition described in (7) at the outlet, whereas at the inlet we use

$$
P_{\mathrm{ar}}(t)=P_{\mathrm{tot}}(0, t)-P_{\mathrm{ext}}, \quad \pi R(0, t) v(0, t)=Q_{\mathrm{ar}}(t),
$$


where $P_{\mathrm{ar}}(t)$ and $Q_{\mathrm{ar}}(t)$ are the arterial pressure and the arterial flow, respectively. We obtain the following system of equations:

$$
\begin{aligned}
& 2\left(\partial_{t} \Phi, \tilde{\Phi}\right)+\pi\left(\partial_{s}(R v), \tilde{\Phi}, \xi(R)\right)=0, \\
& \begin{aligned}
\pi \rho\left(\partial_{t} v, \tilde{v}\right)+\tilde{a}\left(\tilde{v}, v, \frac{v}{R}\right)-\pi\left(\partial_{s}(R \tilde{v}), \Phi \xi(R)\right)+c(R ; v, \tilde{v})+\rho K_{r}\left(\frac{v}{R}, \frac{\tilde{v}}{R}\right) \\
\quad=-\pi \tilde{v}(L) R(L)\left(P_{c}+R_{c} \pi R(L) v(L)\right)+\pi \tilde{v}(0) R(0) P_{\mathrm{ar}},
\end{aligned} \\
& R=r(\Phi), \\
& \pi R(0) v(0)=Q_{\mathrm{ar}}, \\
& C_{c} \frac{\mathrm{d}}{\mathrm{d} t} P_{c}+\frac{P_{c}}{R_{\mathrm{per}}}=\pi R(L) v(L) .
\end{aligned}
$$

Note that a similar energy identity to the one given in Theorem 2 can be derived for this system, as we state below.

Theorem 14 Any smooth solution of System (60) satisfies the conservation property

$$
\frac{\mathrm{d}}{\mathrm{d} t}\left(\mathcal{E}_{a r}+\mathcal{E}_{w}\right)+\mathcal{D}_{a r}+\mathcal{D}_{w}=P_{a r} Q_{a r}
$$

where,

$$
\mathcal{E}_{a r}=\int_{0}^{L} \Phi^{2} \mathrm{~d} s+\frac{\pi \rho}{2} \int_{0}^{L} v^{2} \mathrm{~d} s, \quad \mathcal{E}_{w}=\frac{C_{c}}{2} P_{c}^{2},
$$

and,

$$
\mathcal{D}_{a r}=\rho K_{r} \int_{0}^{L} \frac{v^{2}}{R^{2}} \mathrm{~d} s+\frac{\sqrt{\pi} \Gamma}{R_{0}^{2}} \int_{0}^{L} \frac{1}{R}\left(\partial_{s}(R v)\right)^{2} \mathrm{~d} s, \quad \mathcal{D}_{w}=\frac{P_{c}^{2}}{R_{p e r}}+R_{c}(\pi R(L) v(L))^{2} .
$$

Note that System (14) can be easily used with or without coupling with the reduced heart model. Hence, we consider two cases:

- Case 1: Imposed inlet flux;

In this case the arterial pressure $P_{\mathrm{ar}}$ is considered as a new unknown, namely a Lagrange multiplier for the constraint $\pi R(0) \nu(0)=Q_{a r}$.

- Case 2: Coupling with the reduced heart model.

System (60) should then be completed with Eqs. (10), (14) and (17), that describe the reduced-order cardiac mechanics, the microscopic actin-myosin binding model and the valve model, respectively. Note that in this model $Q_{\mathrm{ar}}$ is an unknown that can be straightforwardly substituted in (17) using the relation $Q_{\mathrm{ar}}=\pi R(0) v(0)$.

\section{Discretization}

Time scheme for the blood flow model

In order to obtain the time discretization of the scheme, we assume a given sequence of time instants $\left\{t^{n}\right\}_{n \in \mathbb{N}}$ such that $t^{n+1}>t^{n}$ and we define the time step as $\Delta t_{n}:=$ $t^{n+1}-t^{n}$. Moreover, we define the half time sequence as $t^{n+\frac{1}{2}}:=t^{n}+\Delta t_{n} / 2$. The scheme proposed below is formally an implicit second-order time discretization scheme: we consider System (48), we rewrite it at time $t^{n+1 / 2}$ and we approximate all the terms using a centered finite difference, i. e. for every $n$

$$
\partial_{t} v\left(t^{n+\frac{1}{2}}\right) \sim \frac{v^{n+1}-v^{n}}{\Delta t_{n}}, \quad \partial_{t} \Phi\left(t^{n+\frac{1}{2}}\right) \sim \frac{\Phi^{n+1}-\Phi^{n}}{\Delta t_{n}}, \quad \frac{\mathrm{d}}{\mathrm{d} t} P_{c} \sim \frac{P_{c}^{n+1}-P_{c}^{n}}{\Delta t_{n}}
$$


and

$$
\begin{aligned}
v\left(t^{n+\frac{1}{2}}\right) & \sim \frac{v^{n+1}+v^{n}}{2}=: v^{n+\frac{1}{2}}, \quad \Phi\left(t^{n+\frac{1}{2}}\right) \sim \frac{\Phi^{n+1}+\Phi^{n}}{2}=: \Phi^{n+\frac{1}{2}}, \\
P_{c}\left(t^{n+\frac{1}{2}}\right) & \sim \frac{P_{c}^{n+1}+P_{c}^{n}}{2}=: P_{c}^{n+\frac{1}{2}} .
\end{aligned}
$$

Moreover, as in [36], we introduce some intermediate unknowns, i.e. $R^{n+\frac{1}{2} \sharp}$ and $\xi^{n+\frac{1}{2} \sharp}$, as follows :

$$
R\left(t^{n+\frac{1}{2}}\right) \sim R^{n+\frac{1}{2} \sharp}:=r\left(\Phi^{n+\frac{1}{2}}\right), \quad \xi\left(t^{n+\frac{1}{2}}\right) \sim \xi^{n+\frac{1}{2} \sharp}:=\xi\left(R^{n+\frac{1}{2} \sharp}\right) .
$$

With these considerations, we obtain the following semi-discrete problem

$$
\left\{\begin{array}{l}
2\left(\frac{\Phi^{n+1}-\Phi^{n}}{\Delta t_{n}}, \tilde{\Phi}\right)+\pi\left(\partial_{s}\left(R^{n+\frac{1}{2} \sharp} v^{n+\frac{1}{2}}\right), \tilde{\Phi} \xi^{n+\frac{1}{2}}\right)=0, \\
\pi \rho\left(\frac{v^{n+1}-v^{n}}{\Delta t_{n}}, \tilde{v}\right)+\tilde{a}\left(\tilde{v}, v^{n+\frac{1}{2}}, \frac{v^{n+\frac{1}{2}}}{R^{n+\frac{1}{2} \sharp}}\right)-\pi\left(\partial_{s}\left(R^{n+\frac{1}{2} \sharp} \tilde{v}\right), \Phi^{n+\frac{1}{2}} \xi^{n+\frac{1}{2}}\right) \\
\quad+c\left(R^{n+\frac{1}{2} \sharp} ; v^{n+\frac{1}{2}}, \tilde{v}\right)+\rho K_{r}\left(\frac{v^{n+\frac{1}{2}}}{R^{n+\frac{1}{2} \sharp}}, \frac{\tilde{v}}{R^{n+\frac{1}{2} \sharp}}\right)=\pi \tilde{v}(0) R^{n+\frac{1}{2} \sharp}(0) P_{\mathrm{ar}}^{n+\frac{1}{2}} \\
\quad-\pi \tilde{v}(L) R^{n+\frac{1}{2} \sharp}(L)\left(P_{c}^{n+\frac{1}{2}}+R_{c} \pi R^{n+\frac{1}{2} \sharp}(L) v^{n+\frac{1}{2}}(L)\right), \\
R^{n+\frac{1}{2} \sharp}=r\left(\Phi^{n+\frac{1}{2}}\right), \\
\xi^{n+\frac{1}{2} \sharp}=\xi\left(R^{n+\frac{1}{2} \sharp}\right), \\
\pi R^{n+\frac{1}{2} \sharp}(0) v^{n+\frac{1}{2}}(0)=Q_{\mathrm{ar}}^{n+\frac{1}{2} \sharp}, \\
C_{c} \frac{P_{c}^{n+1}-P_{c}^{n}}{\Delta t_{n}}+\frac{P_{c}^{n+\frac{1}{2}}}{R_{\mathrm{per}}}=\pi R^{n+\frac{1}{2} \sharp}(L) v^{n+\frac{1}{2}}(L) .
\end{array}\right.
$$

In (62) the terms $Q_{\mathrm{ar}}^{n+\frac{1}{2} \sharp}$ and $P_{\mathrm{ar}}^{n+\frac{1}{2}}$ are not completely characterized yet. These quantities appear below when considering the time discretization of the valve model.

Once (62) is obtained, we have to check if the semi-discrete scheme preserves the total energy, as given in "Outflow conditions, inflow conditions and energy relation" section for the continuous domain. This is the purpose of the following Section.

\section{Semi-discrete energy relation}

Energy preservation can be proven rather simply by following the strategy performed at the continuous level. We substitute the test functions with the proper variables as explained in "Variational formulation in $(\Phi, v)$ " section. More precisely, we set

$$
\tilde{v}=v^{n+\frac{1}{2}}, \quad \tilde{\Phi}=\Phi^{n+\frac{1}{2}} .
$$

We observe that

$$
\left(\frac{\Phi^{n+1}-\Phi^{n}}{\Delta t_{n}}, \Phi^{n+\frac{1}{2}}\right)=\frac{1}{2} \frac{\left\|\Phi^{n+1}\right\|^{2}-\left\|\Phi^{n}\right\|^{2}}{\Delta t_{n}}
$$

where, by definition $\|u\|^{2}=(u, u)$ is the $L^{2}(0, L)$-norm. Identically, we have

$$
\left(\frac{v^{n+1}-v^{n}}{\Delta t_{n}}, v^{n+\frac{1}{2}}\right)=\frac{1}{2} \frac{\left\|v^{n+1}\right\|^{2}-\left\|v^{n}\right\|^{2}}{\Delta t_{n}} .
$$


The first two equations of System (62) become

$$
\left\{\begin{array}{c}
\frac{\left\|\Phi^{n+1}\right\|^{2}-\left\|\Phi^{n}\right\|^{2}}{\Delta t_{n}}+\pi\left(\partial_{s}\left(R^{n+\frac{1}{2} \sharp} v^{n+\frac{1}{2}}\right), \Phi^{n+\frac{1}{2}} \xi^{n+\frac{1}{2}}\right)=0, \\
\frac{\pi \rho}{2} \frac{\left\|v^{n+1}\right\|^{2}-\left\|v^{n}\right\|^{2}}{\Delta t_{n}}+\tilde{a}\left(v^{n+\frac{1}{2}}, v^{n+\frac{1}{2}}, \frac{v^{n+\frac{1}{2}}}{R^{n+\frac{1}{2} \sharp}}\right) \\
-\pi\left(\partial_{s}\left(R^{n+\frac{1}{2} \sharp} v^{n+\frac{1}{2}}\right), \Phi^{n+\frac{1}{2}} \xi^{n+\frac{1}{2}}\right)+c\left(R^{n+\frac{1}{2} \sharp} ; v^{n+\frac{1}{2}}, v^{n+\frac{1}{2}}\right) \\
+\rho K_{r}\left(\frac{v^{n+\frac{1}{2}}}{R^{n+\frac{1}{2} \sharp}}, \frac{v^{n+\frac{1}{2}}}{R^{n+\frac{1}{2} \sharp}}\right)=\text { boundary terms. }
\end{array}\right.
$$

Now, we can observe that the second term of the continuity equation and the third term of the momentum equation are equal (up to a change of sign). Thus, the latter can be substituted with $\left(\left\|\Phi^{n+1}\right\|^{2}-\left\|\Phi^{n}\right\|^{2}\right) / \Delta t_{n}$, which gives

$$
\begin{aligned}
& \frac{\pi \rho}{2} \frac{\left\|v^{n+1}\right\|^{2}-\left\|v^{n}\right\|^{2}}{\Delta t_{n}}+\frac{\left\|\Phi^{n+1}\right\|^{2}-\left\|\Phi^{n}\right\|^{2}}{\Delta t_{n}}+\tilde{a}\left(v^{n+\frac{1}{2}}, v^{n+\frac{1}{2}}, \frac{v^{n+\frac{1}{2}}}{R^{n+\frac{1}{2} \sharp}}\right) \\
& \quad+c\left(R^{n+\frac{1}{2} \sharp} ; v^{n+\frac{1}{2}}, v^{n+\frac{1}{2}}\right)+\rho K_{r}\left(\frac{v^{n+\frac{1}{2}}}{R^{n+\frac{1}{2} \sharp}}, \frac{v^{n+\frac{1}{2}}}{R^{n+\frac{1}{2} \sharp}}\right)=\text { boundary terms. }
\end{aligned}
$$

The equation above can be further simplified by noticing that

$$
\tilde{a}\left(v^{n+\frac{1}{2}}, v^{n+\frac{1}{2}}, v^{n+\frac{1}{2}} / R^{n+\frac{1}{2} \sharp}\right)=0,
$$

thanks to (39). Then, we can define,

$$
\mathcal{E}_{\mathrm{ar}}^{n}=\frac{\pi \rho}{2}\left\|v^{n}\right\|^{2}+\left\|\Phi^{n}\right\|^{2}, \quad \mathcal{E}_{\mathrm{w}}^{n}=\frac{C_{c}}{2}\left(P_{c}^{n}\right)^{2}
$$

and,

$$
\begin{gathered}
\mathcal{D}_{\mathrm{ar}}^{n+\frac{1}{2}}=\rho K_{r}\left\|\frac{v^{n+\frac{1}{2}}}{R^{n+\frac{1}{2} \sharp}}\right\|^{2}+\frac{\sqrt{\pi} \Gamma}{R_{0}^{2}}\left\|\frac{1}{\sqrt{R^{n+\frac{1}{2} \sharp}}} \partial_{s}\left(R^{n+\frac{1}{2} \sharp} v^{n+\frac{1}{2}}\right)\right\|^{2}, \\
\mathcal{D}_{\mathrm{w}}^{n+\frac{1}{2}}=\frac{\left(P_{c}^{n+\frac{1}{2}}\right)^{2}}{R_{\text {per }}}+R_{c}\left(\pi R^{n+\frac{1}{2} \sharp}(L) v^{n+\frac{1}{2}}(L)\right)^{2},
\end{gathered}
$$

and prove, by using a telescopic sum and by adequately dealing with the boundary terms, a semi-discrete equivalent form of (61), showing that Theorem 14 has a counterpart at the semi-discrete level.

Theorem 15 Any solution of System (62) satisfies the following conservation property for all $n \in \mathbb{N}$,

$$
\frac{\mathcal{E}_{a r}^{n+1}-\mathcal{E}_{a r}^{n}}{\Delta t_{n}}+\frac{\mathcal{E}_{w}^{n+1}-\mathcal{E}_{w}^{n}}{\Delta t_{n}}+\mathcal{D}_{a r}^{n+\frac{1}{2}}+\mathcal{D}_{w}^{n+\frac{1}{2}}=P_{a r}^{n+\frac{1}{2}} Q_{a r}^{n+\frac{1}{2} \sharp}
$$

Note that the energy relation obtained in Theorem 15 holds with a time step that may vary between each iteration. This property is fundamental since, in practice, cardiac models often adapt the time step to deal with the abrupt changes of phase due the opening and closure of the aortic valve.

\section{Time scheme for the heart model}

The discretization of the heart model was performed following a similar approach to the one described in the previous section. This allows to obtain, as for the arterial model, 
a discrete equivalent of the conservation property described in Section . The time discretization method used in [36] is adapted to the zero-dimensional formulation of the cavity with the additional introduction of the so-called Hilber-Hughes-Taylor (HHT) Method scheme proposed in [44] for the treatment of kinetics variables and inertial terms. The discrete velocity $\dot{y}^{n}$ and the displacement $y^{n}$ are related by the use of an auxiliary variable $\ddot{y}^{n}$-that stands for an approximation of the acceleration. We proceed discretizing the equations of System (10) at time $t^{n+\frac{1}{2}}$ except for the ventricular, atrial and arterial pressures that are interpolated using a $\theta$-scheme $\left(P_{v}^{n+\theta}=(1-\theta) P_{v}^{n}+\theta P_{v}^{n+1}, \theta \in[0,1]\right)$. Deviations from the classical implicit mid-point scheme used in "Time scheme for the blood flow model" section are introduced to generate controlled numerical dissipation terms that damp out purely numerical high frequencies modes that cannot be properly resolved with a finite time step-see also "Spurious high frequencies filtering" section. The HHT kinematics equations are

$$
\left\{\begin{array}{l}
\frac{\dot{y}^{n+1}-\dot{y}^{n}}{\Delta t_{n}}=\ddot{y}^{n+\frac{1}{2}}+\alpha \Delta t_{n} \frac{\ddot{y}^{n+1}-\ddot{y}^{n}}{\Delta t_{n}}, \\
\frac{y^{n+1}-y^{n}}{\Delta t_{n}}=\dot{y}^{n+\frac{1}{2}}+\frac{\alpha^{2}}{4} \Delta t_{n}^{2} \frac{\ddot{y}^{n+1}-\ddot{y}^{n}}{\Delta t_{n}} .
\end{array}\right.
$$

and the dynamics equations then read

$$
\left\{\begin{array}{c}
\rho_{0}\left|\Omega_{0}\right|\left(\frac{\dot{y}^{n+1}-\dot{y}^{n}}{\Delta t_{n}}\right)+\frac{\left|\Omega_{0}\right|}{R_{0}} k_{s}\left(\frac{y^{n+\frac{1}{2}}}{R_{0}}-e_{c}^{n+\frac{1}{2}}\right) \\
\quad+\Lambda_{\mathrm{hyp}}^{n+\frac{1}{2} \sharp}+\mathcal{W}_{v}\left(y^{n+\frac{1}{2}}, \frac{y^{n+1}-y^{n}}{\Delta t_{n}}\right)=P_{v}^{n+\theta} S^{n+\frac{1}{2} \sharp}, \\
\mu \frac{e_{c}^{n+1}-e_{c}^{n}}{\Delta t_{n}}-k_{s}\left(\frac{y^{n+\frac{1}{2}}}{R_{0}}-e_{c}^{n+\frac{1}{2}}\right)=-\tau_{c}^{n+\frac{1}{2} \sharp},
\end{array}\right.
$$

where we use the adapted [45] energy-preserving non-linear choice

$$
\Lambda_{\text {hyp }}^{n+\frac{1}{2} \sharp}= \begin{cases}\frac{\mathcal{W}_{p}\left(y^{n+1}\right)-\mathcal{W}_{p}\left(y^{n}\right)}{y^{n+1}-y^{n}} & \text { if } y^{n+1} \neq y^{n}, \\ \frac{\partial \mathcal{W}_{p}}{\partial y}\left(y^{n+\frac{1}{2}}\right) & \text { if } y^{n+1}=y^{n}=y^{n+\frac{1}{2}},\end{cases}
$$

and additionally

$$
S^{n+\frac{1}{2} \sharp}= \begin{cases}\frac{V\left(y^{n+1}\right)-V\left(y^{n}\right)}{y^{n+1}-y^{n}} & \text { if } y^{n+1} \neq y^{n}, \\ \frac{\partial V}{\partial y}\left(y^{n+\frac{1}{2}}\right) & \text { if } y^{n+1}=y^{n}=y^{n+\frac{1}{2}} .\end{cases}
$$

Note that in practice, the expressions for $y^{n+1} \neq y^{n}$ are implemented using a series development of $\mathcal{W}_{p}$ and $V$, respectively, to avoid numerical rounding errors. Further, $\tau_{c}^{n+\frac{1}{2} \sharp}$ is computed from the already proposed energy-balanced time discretization of System (14) and (16) [36], namely

$$
\begin{aligned}
& \tau_{c}^{n+\frac{1}{2} \sharp}=\sqrt{k_{c}^{n+1}} \lambda_{c}^{n+\frac{1}{2}} \text { with } \\
& \left\{\begin{array}{l}
\frac{k_{c}^{n+1}-k_{c}^{n}}{\Delta t_{n}}=-\left(\left|v^{n+1}\right|+\alpha\left|\frac{e_{c}^{n+1}-e_{c}^{n}}{\Delta t_{n}}\right|\right) k_{c}^{n+1} \\
+n_{0}\left(e_{c}^{n}\right) k_{0}\left|v^{n+1}\right|_{+} \\
\frac{\lambda_{c}^{n+1}-\lambda_{c}^{n}}{\Delta t_{n}}=-\frac{1}{2}\left(\left|v^{n+1}\right|+\alpha\left|\frac{e_{c}^{n+1}-e_{c}^{n}}{\Delta t_{n}}\right|\right) \lambda_{c}^{n+\frac{1}{2}} \\
+\frac{n_{0}\left(e_{c}^{n}\right)}{\sqrt{k_{c}^{n+1}}}\left(\sigma_{0}-\frac{k_{0} \lambda_{c}^{n+\frac{1}{2}}}{2 \sqrt{k_{c}^{n+1}}}\right)\left|v^{n+1}\right|_{+}+\sqrt{k_{c}^{n+1}} \frac{e_{c}^{n+1}-e_{c}^{n}}{\Delta t_{n}} .
\end{array}\right.
\end{aligned}
$$


Since the function $n_{0}(\cdot)$ has maximum value 1 , it is straightforward to prove [36], from backward Euler time discretization, the a priori bound $0<k_{c}^{n} \leq k_{0}$ for all $n \geq 1$ if it is satisfied at $n=0$. Moreover, the time discretization of the variable $\lambda^{n}$ is consistent with the dynamics of $\lambda_{c}=\tau_{c} / \sqrt{k_{c}}$ given in Remark 5 .

In order to retrieve a discrete equivalent of the discrete energy balance obtained in Section, we multiply the second equation of System (68) by $\left(y^{n+1}-y^{n}\right) / \Delta t$ and the third one by $\left|\Omega_{0}\right|\left(e_{c}^{n+1}-e_{c}^{n}\right) / \Delta t_{n}$, then we sum, obtaining

$$
\begin{gathered}
\frac{\mathcal{E}_{\mathrm{hr}}^{n+1}-\mathcal{E}_{\mathrm{hr}}^{n}}{\Delta t_{n}}+\frac{\mathcal{E}_{\mathrm{hr}, \text { num }}^{n+1}-\mathcal{E}_{\mathrm{hr}, \text { num }}^{n}}{\Delta t_{n}}+\mathcal{D}_{\mathrm{hr}}^{n+\frac{1}{2}}+\mathcal{D}_{\mathrm{hr}, \text { num }}^{n+\frac{1}{\sharp}} \\
=-\left|\Omega_{0}\right| \frac{e_{c}^{n+1}-e_{c}^{n}}{\Delta t_{n}} \tau_{c}^{n+\frac{1}{2} \sharp}+P_{v}^{n+\theta} \frac{V\left(y^{n+1}\right)-V\left(y^{n}\right)}{\Delta t_{n}},
\end{gathered}
$$

where we have introduced the discrete energy

$$
\mathcal{E}_{\mathrm{hr}}^{n}:=\frac{\rho_{0}\left|\Omega_{0}\right|}{2}\left(\dot{y}^{n}\right)^{2}+\mathcal{W}_{p}\left(y^{n}\right)+\frac{\left|\Omega_{0}\right| k_{s}}{2}\left(\frac{y^{n}}{R_{0}}-e_{c}^{n}\right)^{2},
$$

the discrete dissipation term

$$
\mathcal{D}_{\mathrm{hr}}^{n+\frac{1}{2}}:=\mathcal{W}_{\nu}\left(y^{n+\frac{1}{2}}, \frac{y^{n+1}-y^{n}}{\Delta t_{n}}\right) \frac{y^{n+1}-y^{n}}{\Delta t_{n}}+\mu\left|\Omega_{0}\right|\left(\frac{e_{c}^{n+1}-e_{c}^{n}}{\Delta t_{n}}\right)^{2},
$$

as well as a numerical stored energy

$$
\mathcal{E}_{\text {hr,num }}^{n}:=\alpha^{2} \Delta t_{n}^{2} \frac{\rho_{0}\left|\Omega_{0}\right|}{8}\left(\ddot{y}^{n}\right)^{2}
$$

and a numerical dissipated energy

$$
\mathcal{D}_{\mathrm{hr}, \text { num }}^{n+\frac{1}{2} \sharp}:=\alpha^{3} \Delta t_{n}^{3} \frac{\rho_{0}\left|\Omega_{0}\right|}{4}\left(\frac{\ddot{y}^{n+1}-\ddot{y}^{n}}{\Delta t_{n}}\right)^{2},
$$

which scale both with powers of $\Delta t_{n}$. Moreover, multiplying the second equation of (69) by $\left|\Omega_{0}\right| \lambda_{c}^{n+\frac{1}{2}}$ we obtain

$$
\begin{aligned}
\frac{\mathcal{E}_{c}^{n+1}-\mathcal{E}_{c}^{n}}{\Delta t_{n}}+\mathcal{D}_{c}^{n+\frac{1}{2}}= & \frac{\left|\Omega_{0}\right| n_{0}\left(e_{c}^{n}\right)}{\sqrt{k_{c}^{n+1}}}\left(\sigma_{0} \lambda_{c}^{n+\frac{1}{2}}-\frac{k_{0}\left(\lambda_{c}^{n+\frac{1}{2}}\right)^{2}}{2 \sqrt{k_{c}^{n+1}}}\right)\left|v^{n+1}\right|_{+} \\
& +\left|\Omega_{0}\right| \frac{e_{c}^{n+1}-e_{c}^{n}}{\Delta t_{n}} \tau_{c}^{n+\frac{1}{2} \sharp}
\end{aligned}
$$

with

$$
\mathcal{E}_{c}^{n}:=\frac{\left|\Omega_{0}\right|}{2}\left(\lambda_{c}^{n}\right)^{2}, \quad \mathcal{D}_{c}^{n+\frac{1}{2}}:=\left(\left|v^{n+1}\right|+\alpha\left|\frac{e_{c}^{n+1}-e_{c}^{n}}{\Delta t_{n}}\right|\right) \frac{\left|\Omega_{0}\right|}{2}\left(\lambda_{c}^{n+\frac{1}{2}}\right)^{2} .
$$

Note that (71), (72) and (76) are the time-discrete equivalent of (11), (12) and (13) respectively. Summing (70) and (75), we obtain the following result.

Theorem 16 Any solution of System (68) and System (69) satisfies the following conservation property for all $n \in \mathbb{N}$ :

$$
\begin{aligned}
& \frac{\mathcal{E}_{h r}^{n+1}-\mathcal{E}_{h r}^{n}}{\Delta t_{n}}+\frac{\mathcal{E}_{c}^{n+1}-\mathcal{E}_{c}^{n}}{\Delta t_{n}}+\mathcal{D}_{h r}^{n+\frac{1}{2}}+\mathcal{D}_{c}^{n+\frac{1}{2}}=P_{v}^{n+\theta} \frac{V\left(y^{n+1}\right)-V\left(y^{n}\right)}{\Delta t_{n}} \\
& +\frac{\left|\Omega_{0}\right| n_{0}\left(e_{c}^{n}\right)}{\sqrt{k_{c}^{n+1}}}\left(\sigma_{0} \lambda_{c}^{n+\frac{1}{2}}-\frac{k_{0}\left(\lambda_{c}^{n+\frac{1}{2}}\right)^{2}}{2 \sqrt{k_{c}^{n+1}}}\right)\left|v^{n+1}\right|_{+} .
\end{aligned}
$$


Time scheme for the valves and the energy relation for the complete semi-discrete system

The discretization strategy applied for the valve formulation is the same that we showed for the arterial and ventricular model. In particular, we use an implicit mid-point rule, i.e.

$$
-\frac{\mathrm{d}}{\mathrm{d} t}(v(y))=Q_{v} \sim-\frac{\left.V\left(y^{n+1}\right)-V\left(y^{n}\right)\right)}{\Delta t_{n}}
$$

and

$$
Q_{\mathrm{ar}} \sim Q_{\mathrm{ar}}^{n+\frac{1}{2} \sharp}=\pi R^{n+\frac{1}{2} \sharp} v^{n+\frac{1}{2}}, \quad \frac{\left|P_{v}-P_{\mathrm{ar}}\right|_{+}}{K_{\mathrm{ar}}} \sim \frac{\left|P_{v}^{n+\theta}-P_{\mathrm{ar}}^{n+\frac{1}{2}}\right|_{+}}{K_{\mathrm{ar}}} .
$$

System (17) is then discretized as follows:

$$
\left\{\begin{array}{l}
C_{\mathrm{mi}} \frac{P_{v}^{n+1}-P_{v}^{n}}{\Delta t_{n}}+\frac{\left|P_{v}^{n+\theta}-P_{\mathrm{ar}}^{n+\frac{1}{2}}\right|_{+}}{K_{\mathrm{ar}}} \\
\quad+\frac{\left|P_{v}^{n+\theta}-P_{\mathrm{at}}^{n+\frac{1}{2}}\right|_{+}}{K_{\mathrm{iso}}}-\frac{\left|P_{\mathrm{at}}^{n+\frac{1}{2}}-P_{v}^{n+\theta}\right|_{+}}{K_{\mathrm{at}}}=-\frac{\left.V\left(y^{n+1}\right)-V\left(y^{n}\right)\right)}{\Delta t_{n}}, \\
C_{\mathrm{ar}} \frac{P_{\mathrm{ar}}^{n+1}-P_{\mathrm{ar}}^{n}}{\Delta t_{n}}-\frac{\left|P_{v}^{n+\theta}-P_{\mathrm{ar}}^{n+\frac{1}{2}}\right|_{+}}{K_{\mathrm{ar}}}=-Q_{\mathrm{ar}}^{n+\frac{1}{2} \sharp} .
\end{array}\right.
$$

To obtain an energy identity we multiply the first equation of System (78) by $P_{v}^{n+\theta}$ and the second one by $P_{\mathrm{ar}}^{n+\frac{1}{2}}$, then we sum the two equations. The discrete conservation property reads

$$
\begin{aligned}
& \frac{\mathcal{E}_{v}^{n+1}-\mathcal{E}_{v}^{n}}{\Delta t_{n}}+\mathcal{D}_{v}^{n+\theta}+\mathcal{D}_{v, \text { num }}^{n+\frac{1}{2} \sharp}=\frac{\left|P_{\mathrm{at}}^{n+\frac{1}{2}}-P_{v}^{n+\theta}\right|_{+} P_{\mathrm{at}}^{n+\frac{1}{2}}}{K_{\mathrm{at}}} \\
& -\frac{\left|P_{v}^{n+\theta}-P_{\mathrm{at}}^{n+\frac{1}{2}}\right|_{+} P_{\mathrm{at}}^{n+\frac{1}{2}}}{K_{\text {iso }}}-P_{v}^{n+\theta} \frac{V\left(y^{n+1}\right)-V\left(y^{n}\right)}{\Delta t_{n}}-P_{\mathrm{ar}}^{n+\frac{1}{2}} Q_{\mathrm{ar}}^{n+\frac{1}{2} \sharp},
\end{aligned}
$$

where the discrete energy for the valve model reads

$$
\frac{\mathcal{E}_{v}^{n+1}-\mathcal{E}_{v}^{n}}{\Delta t_{n}}=\frac{C_{\mathrm{mi}}}{2} \frac{\left(P_{v}^{n+1}\right)^{2}-\left(P_{v}^{n}\right)^{2}}{\Delta t_{n}}+\frac{C_{\mathrm{ar}}}{2} \frac{\left(P_{\mathrm{ar}}^{n+1}\right)^{2}-\left(P_{\mathrm{ar}}^{n}\right)^{2}}{\Delta t_{n}},
$$

the dissipation is

$$
\mathcal{D}_{v}^{n+\theta}=\frac{\left|P_{v}^{n+\theta}-P_{\mathrm{ar}}^{n+\frac{1}{2}}\right|_{+}^{2}}{K_{\mathrm{ar}}}+\frac{\left|P_{v}^{n+\theta}-P_{\mathrm{at}}^{n+\frac{1}{2}}\right|_{+}^{2}}{K_{\mathrm{iso}}}+\frac{\left|P_{\mathrm{at}}^{n+\frac{1}{2}}-P_{v}^{n+\theta}\right|_{+}^{2}}{K_{\mathrm{at}}},
$$

and the numerical dissipation reads

$$
\mathcal{D}_{v, \text { num }}^{n+\frac{1}{2} \sharp}=\left(\theta-\frac{1}{2}\right) C_{\mathrm{mi}} \Delta t_{n}\left(\frac{P_{v}^{n+1}-P_{v}^{n}}{\Delta t_{n}}\right)^{2} .
$$

\section{The discrete energy relation for the global system}

Finally, summing Eqs. [(64), (77) and (79)], we obtain the semi-discrete conservation property for the global system. 
Corollary 17 Any solution of System (62), (68),(69) and (78) satisfies the following conservation property for all $n \in \mathbb{N}$,

$$
\begin{aligned}
& \frac{\mathcal{E}_{a r}^{n+1}-\mathcal{E}_{a r}^{n}}{\Delta t_{n}}+\frac{\mathcal{E}_{w}^{n+1}-\mathcal{E}_{w}^{n}}{\Delta t_{n}}+\frac{\mathcal{E}_{h r}^{n+1}-\mathcal{E}_{h r}^{n}}{\Delta t_{n}}+\frac{\mathcal{E}_{h r, n u m}^{n+1}-\mathcal{E}_{h r, n u m}^{n}}{\Delta t_{n}}+\frac{\mathcal{E}_{v}^{n+1}-\mathcal{E}_{v}^{n}}{\Delta t_{n}} \\
& \quad+\mathcal{D}_{w}^{n+\frac{1}{2}}+\mathcal{D}_{h r}^{n+\frac{1}{2}}+\mathcal{D}_{h r, n u m}^{n+\frac{1}{2} \sharp}+\mathcal{D}_{a r}^{n+\frac{1}{2}}+\mathcal{D}_{v}^{n+\frac{1}{2}}+\mathcal{D}_{v, n u m}^{n+\frac{1}{2} \sharp} \\
& =\frac{\left|\Omega_{0}\right| n_{0}\left(e_{c}^{n}\right)}{\sqrt{k_{c}^{n+1}}}\left(\sigma_{0} \lambda_{c}^{n+\frac{1}{2}}-\frac{k_{0}\left(\lambda_{c}^{n+\frac{1}{2}}\right)^{2}}{2 \sqrt{k_{c}^{n+1}}}\right)\left|v^{n+1}\right|_{+} \\
& \quad+\frac{\left|P_{a t}^{n+\frac{1}{2}}-P_{v}^{n+\theta}\right|_{+} P_{a t}^{n+\frac{1}{2}}}{K_{a t}}-\frac{\left|P_{v}^{n+\theta}-P_{a t}^{n+\frac{1}{2}}\right|_{+} P_{a t}^{n+\frac{1}{2}}}{K_{i s o}} .
\end{aligned}
$$

Corollary 17 shows that without a source term, i.e when $v^{n+1}$ and $P_{\text {at }}^{n+\frac{1}{2}}$ vanish, the energy - that is a norm for the solution-is decaying. This is the expected stability property of a robust time discretization.

\section{Space discretization of the blood flow model}

The space discretization is rather simple and does not represent a main issue for the global formulation. However, there are some terms, in the aortic model formulation, that have to be treated carefully when choosing the space discretization method. This is the reason why, in this section, we will only present the space discretization of the arterial model without dealing with how the total discretization of the aorta model couples with the other elements of the model. Indeed, these couplings are straightforward from what has been already explained.

First, we introduce a finite dimensional subspace of $H^{1}(0, L)$ of continuous functions that is denoted by $V_{h}$ and we assume an interpolation operator $\mathcal{I}_{h}: C^{0}([0, L]) \rightarrow V_{h}$ as given. For each $n \in \mathbb{N}$, we look for the solutions $\left(\Phi_{h}^{n}, v_{h}^{n}\right) \in V_{h} \times V_{h}$, for all $\left(\tilde{\Phi}_{h}, \tilde{v}_{h}\right) \in V_{h} \times V_{h}$, of

$$
\left\{\begin{array}{l}
2\left(\frac{\Phi_{h}^{n+1}-\Phi_{h}^{n}}{\Delta t_{n}}, \tilde{\Phi}_{h}\right)_{h}+\pi\left(\partial_{s}\left(R_{h}^{n+\frac{1}{2} \sharp} v_{h}^{n+\frac{1}{2}}\right), \tilde{\Phi}_{h} \xi_{h}^{n+\frac{1}{2}}\right)_{h}=0, \\
\pi \rho\left(\frac{v_{h}^{n+1}-v_{h}^{n}}{\Delta t_{n}}, \tilde{v}_{h}\right)_{h}+a_{h}\left(v_{h}^{n+\frac{1}{2}}, R_{h}^{n+\frac{1}{2} \sharp} ; \tilde{v}_{h}\right) \\
-\pi\left(\partial_{s}\left(R_{h}^{n+\frac{1}{2} \sharp} \tilde{v}_{h}\right), \Phi_{h}^{n+\frac{1}{2}} \xi_{h}^{n+\frac{1}{2}}\right)_{h}+c_{h}\left(R_{h}^{n+\frac{1}{2} \sharp}, v_{h}^{n+\frac{1}{2}}, \tilde{v}_{h}\right)+\rho K_{r}\left(u_{h}^{n+\frac{1}{2}}, \tilde{u}_{h}\right)_{h} \\
=\text { boundary terms, } \\
R_{h}^{n+\frac{1}{2} \sharp}=\mathcal{I}_{h} r\left(\Phi_{h}^{n+\frac{1}{2}}\right), \\
\xi_{h}^{n+\frac{1}{2} \sharp}=\mathcal{I}_{h} \xi\left(R_{h}^{n+\frac{1}{2} \sharp}\right), \\
u_{h}^{n+\frac{1}{2}}=\mathcal{I}_{h} \frac{v_{h}^{n+\frac{1}{2}}}{R_{h}^{n+\frac{1}{2} \sharp}} \\
\tilde{u}_{h}=\mathcal{I}_{h} \frac{\tilde{v}_{h}}{R_{h}^{n+\frac{1}{2} \sharp} .}
\end{array}\right.
$$

In the equations above the missing boundary terms are easily deduced from the second equation of System (62) by replacing $R^{n+\frac{1}{2} \sharp}$ by $R_{h}^{n+\frac{1}{2} \sharp}, v$ by $v_{h}$ and $\tilde{v}$ by $\tilde{v}_{h}$. Moreover, we have introduced two other notations:

i. We use the notation $(\cdot, \cdot)_{h}$ to represent an approximation of the scalar product in $L^{2}(0, L)$ by quadrature formulae. In particular $(\cdot, \cdot)_{h}$ is a positive definite bilinear form 
and is equivalent to the $L^{2}(0, L)$-norm in $V_{h}$. In what follows, for any $u_{h} \in V_{h}$ we define $\left\|u_{h}\right\|_{h}^{2}:=\left(u_{h}, u_{h}\right)_{h}$.

ii. The critical point from the energetic point of view in performing the space discretization of our scheme can be found in the definition of the term $a_{h}$. As stated in "Time scheme for the blood flow model" section for the energy conservation, this term has to satisfy the property defined in (39). One way to guarantee this condition is to consider the exact-i.e. we compute the integrals exactly-trilinear form $\tilde{a}$. That is why the form $a_{h}$ is defined by

$$
a_{h}\left(v_{h}^{n+\frac{1}{2}}, R_{h}^{n+\frac{1}{2} \sharp} ; \tilde{v}_{h}\right):=\tilde{a}\left(\tilde{v}_{h}, v_{h}^{n+\frac{1}{2}}, u_{h}^{n+\frac{1}{2}}\right) .
$$

With this definition and thanks to (39), it is possible to check that, for all $\left(v_{h}, R_{h}\right) \in$ $V_{h} \times V_{h}$

$$
a_{h}\left(v_{h}, R_{h} ; v_{h}\right)=\tilde{a}\left(v_{h}, v_{h}, \mathcal{I}_{h} \frac{v_{h}}{R_{h}}\right)=0 .
$$

Even though the introduction of the interpolation operator in the definition of $u_{h}^{n+\frac{1}{2}}$ involved in $a_{h}$ given by (82) seems unnecessary, one can observe from the structure of the trilinear form $\tilde{a}$ that its last argument would not be polynomial without interpolation. This implies that the application of a standard quadrature method (e.g. the Gauss integration) would not give an exact integration property, and therefore the property stated by (83) may be lost.

Using (83), it is straightforward to prove that the energy relation stated in Theorem 15 can be extended to the fully-discrete case. We obtain the following result.

Theorem 18 Any solution of System (62) satisfies the following conservation property for all $n \in \mathbb{N}$

$$
\frac{\mathcal{E}_{h}^{n+1}-\mathcal{E}_{h}^{n}}{\Delta t_{n}}+\mathcal{D}_{h}^{n+\frac{1}{2}}=\tilde{g}\left(v_{h}^{n+\frac{1}{2}}, R_{h}^{n+\frac{1}{2} \sharp}\right),
$$

with

$$
\begin{aligned}
& \mathcal{E}_{h}^{n}=\frac{\pi \rho}{2}\left\|v^{n}\right\|_{h}^{2}+\left\|\Phi^{n}\right\|_{h}^{2} \\
& \mathcal{D}_{h}^{n+\frac{1}{2}}=\rho K_{r}\left\|\mathcal{I}_{h} \frac{v_{h}^{n+\frac{1}{2}}}{R_{h}^{n+\frac{1}{2} \sharp}}\right\|_{h}^{2}+\frac{\sqrt{2} \Gamma}{R_{0}^{2}}\left\|\frac{1}{\sqrt{R_{h}^{n+\frac{1}{2} \sharp}}} \partial_{s}\left(R_{h}^{n+\frac{1}{2} \sharp} v_{h}^{n+\frac{1}{2}}\right)\right\|_{h}^{2} .
\end{aligned}
$$

When using the model described for simulations we divide the domain $[0, L]$ into regular subdomains $\ell_{j}=\left[s_{j}, s_{j+1}\right]$ of fixed length $\Delta s$, such that $s_{j}=j \Delta s$ with $j=\{0,1, \ldots, J\}$. In particular, we define the space $V_{h}$ as

$$
V_{h}=\left\{v_{h} \in C^{0}([0, L]) /\left.\forall j \in\{0,1, \ldots, J\} \in v_{h}\right|_{\ell_{j}} \in \mathbb{P}_{1}\left(\ell_{j}\right)\right\}
$$

and we use $\mathbb{P}_{1}$-finite elements. The space $V_{h}$ is spanned by the Lagrange nodal basis functions $\left\{w_{j}\right\}_{j=0}^{J}$ that satisfy the property $w_{j}\left(s_{i}\right)=\delta_{i j}$. The interpolation operator then reads

$$
\left(\mathcal{I}_{h} v\right)(s)=\sum_{j=0}^{J} v\left(s_{j}\right) w_{j}(s)
$$


We have, for all $\left(v_{h}, u_{h}\right) \in V_{h} \times V_{h}$,

$$
\left(v_{h}, u_{h}\right)_{h}=\frac{\Delta s}{2} v_{h}\left(s_{0}\right) u_{h}\left(s_{0}\right)+\Delta s \sum_{j=1}^{J-1} v_{h}\left(s_{j}\right) u_{h}\left(s_{j}\right)+\frac{\Delta s}{2} v_{h}\left(s_{J}\right) u_{h}\left(s_{J}\right) .
$$

The scalar product $(\cdot, \cdot)_{h}$ is defined using the trapezoidal rule, while the term $\tilde{a}\left(\tilde{v}_{h}, v_{h}, u_{h}\right)$ should be computed exactly to ensure energy preservation. To do so, it is sufficient to use the Simpson quadrature method in each element for the underlying integration, sincethanks to the use of the interpolation operator-the integrand is a second-order polynomial in each element.

\section{Simulations and results}

In order to show the results of our work, we consider three different model settings:

- The uncoupled aortic model is composed by a single 1D straight vessel with a homogeneous circular cross-section representing the upper thoracic aorta and by a Windkessel RCR model that takes into account the impedance and compliance of all the remaining vessels at the periphery. In this test case the inlet blood flow is imposed, as shown in Fig. 4. This configuration allows us to study the behavior of the arterial model alone.

- The uncoupled cardiac model, depicted in Fig. 5, is composed by the reduced cardiac model described in the previous sections and by a lumped parameter model as outlet boundary condition that represents the entire circulation. More precisely, we have reduced the 1D model of the aorta into an $\mathrm{RC}$-model, the parameters of the resulting OD model being the equivalent resistance and compliance of the $1 \mathrm{D}$ vessel combined with the RCR boundary model parameters. The equivalent resistance and compliance of the 1D vessel are obtained by (formulas readapted from [46])

$$
R_{1 \mathrm{D}}=\frac{22 \mu_{b} L}{\pi R_{0}^{4}} \quad \text { and } \quad C_{1 \mathrm{D}}=\frac{3}{2} \frac{\pi R_{0}^{3} L}{E h_{0}},
$$

where $\mu_{b}$ is the blood viscosity. This setting allows to show the outcome of the cardiovascular model when the circulation is represented by a simple Windkessel model.

- The fully coupled model studied in the present work, with the reduced-dimensional model of the heart and the aortic model coupled through a transmission condition which includes the valve as represented in Fig. 2.

The simulation results are divided into two main sections. In "Numerical validation" section we present the numerical validation of the proposed formulation, starting from the comparison between the results obtained using the uncoupled aortic model and the results of other numerical schemes. Then, we verify that the energy relation is indeed satisfied at the numerical level and discuss its beneficial effect on the computations. In "Physiological outcomes of the coupling" section we highlight the importance of the coupling involving two case studies: dicrotic notch and physiological ageing. The first case study is considered to demonstrate the need of having a one dimensional model as an outlet boundary for the heart to capture all the important features of pressure waves in the larger elastic arteries (e.g. the aorta), whereas in the second one we show how the simulations of specific conditions of the circulation (e.g. stiffening of the vessels due to 


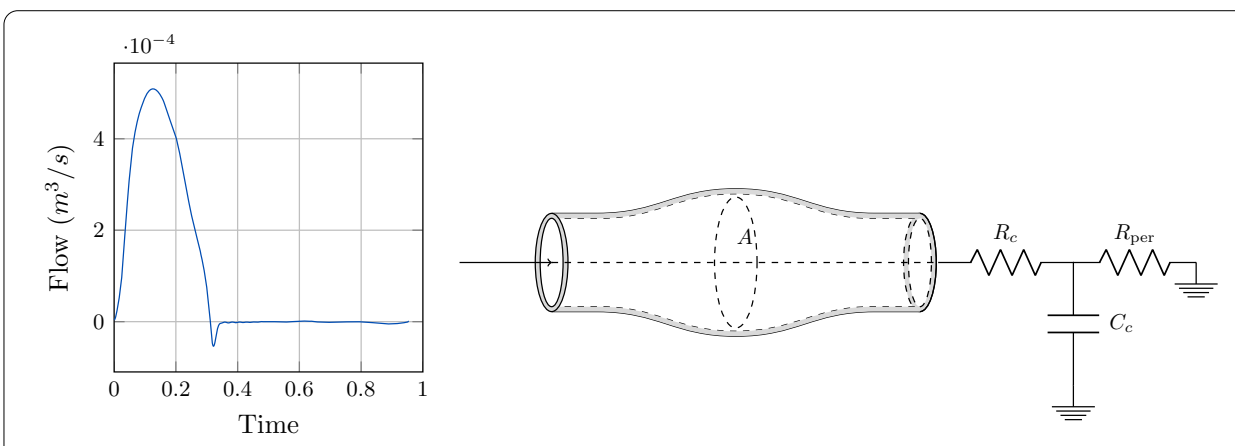

Fig. 4 Scheme of the uncoupled aortic model: single 1D vessel that represents the upper thoracic aorta and a three-element Windkessel model as boundary conditions to represent the remaining part of the circulation

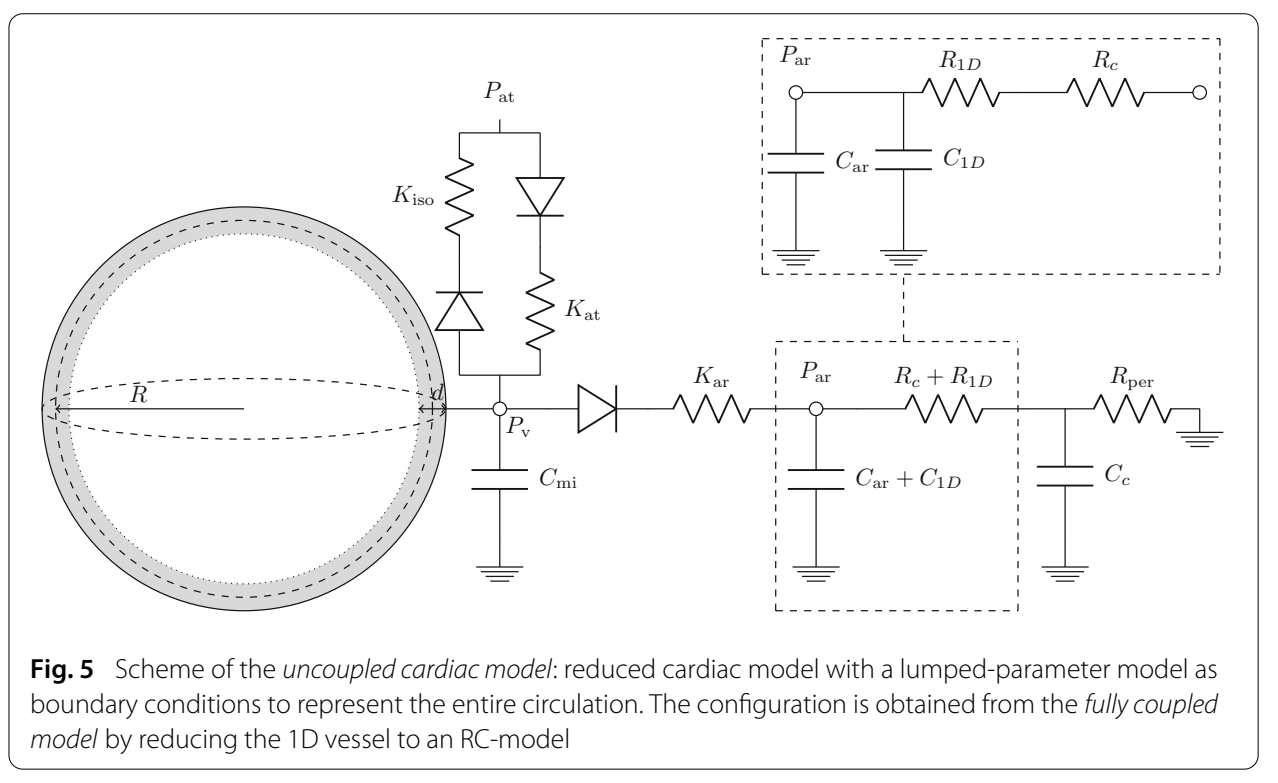

ageing) benefit from the presence of a cardiac model whose behavior depends on the arterial conditions. Both cases reflect the natural interplay between the components of the cardiovascular system [1] .

\section{Numerical validation}

\section{Benchmark for the uncoupled aortic model}

In order to support the validity of the developed method, our scheme is tested in one of the benchmarks presented in [18]. In this work, different numerical schemes are compared for 1D arterial modeling in a set of test cases and they made the results freely available. In the following example we take into account the third benchmark configuration [18], that corresponds to the upper thoracic aorta (setting presented in Fig. 4). For this particular test case the authors considered a single uniform vessel represented by a one-dimensional model, that relies on the classical blood flow equations described in System 1 in the equivalent $(A, u)$ formulation - the state variables are the cross section and the velocityand an RCR model as outlet boundary condition. More precisely, in this benchmark, Eqs. (1), (7) and (8) are solved with $Q_{a r}(t)$ imposed. 
Table 1 Model parameters of the upper thoracic aorta (Readapted from [23])

\begin{tabular}{ll}
\hline Property & Value \\
\hline Length, $\mathrm{L}$ & $24.137 \mathrm{~cm}$ \\
Radius at diastolic pressure, $R_{0}$ & $1.2 \mathrm{~cm}$ \\
Initial flow velocity, $u(s, 0)$ & $0 \mathrm{~m} \mathrm{~s}^{-1}$ \\
Initial pressure, $P(s, 0)$ & $0 \mathrm{~Pa}$ \\
Wall thickness, $h_{0}$ & $1.2 \mathrm{~mm}$ \\
Blood density, $\rho$ & $1060 \mathrm{Kg} \mathrm{m}{ }^{-3}$ \\
Friction parameter, $K_{r}$ & $276.46 \mathrm{mPa} \mathrm{s}$ \\
Wall viscosity, $v$ & $0 \mathrm{mPa} \mathrm{s}$ \\
Velocity profile, $\alpha_{v p}$ & 1 \\
Young's modulus, $E$ & $400.0 \mathrm{kPa}$ \\
Diastolic pressure, $P_{D}$ & $9.46 \mathrm{kPa}$ \\
External pressure, $P_{\text {ext }}$ & $0 \mathrm{~Pa}$ \\
Windkessel resistance, $R_{c}$ & $1.1752 \cdot 10^{7} \mathrm{~Pa} \mathrm{~s} \mathrm{m^{-3 }}$ \\
Windkessel compliance, $C_{c}$ & $1.0163 \cdot 10^{-8} \mathrm{~m}^{3} \mathrm{~Pa}^{-1}$ \\
Windkessel resistance, $R_{\text {per }}$ & $1.1167 \cdot 10^{8} \mathrm{~Pa} \mathrm{~s} \mathrm{~m}^{-3}$ \\
\hline
\end{tabular}

The parameters of the model and the inflow boundary condition used in [18] were taken from [23] and are reported, respectively, in Table 1 and Fig. 4. We considered these same parameters and inlet conditions, as shown in Fig. 6, and we compared our results with those of other numerical schemes available in the datasets of [18]. In Fig. 6 it is possible to observe that the pressure curves obtained by the numerical schemes reported are consistent, hence validates our implementation. This shows that our formulation is consistent with well-known discretizations. At the price of a higher computational costdue to the implicit nature - the proposed scheme has, in addition, the advantage to offer a provably stable numerical coupling with a numerical approximation of a non-linear description of the heart.

\section{Spurious high frequencies filtering}

In the discretization section, we have introduced two parameters $\alpha$ and $\theta$ associated with a controlled artificial viscosity of order $\delta t$ in order to damp possible undesired oscillations (when $\alpha>0$ and $\theta>1 / 2$ ). We emphasize that these oscillations are not due to numerical instability, but to energy exchanges between model compartments introduced by the time discretization procedure, in particular the conservative part involving mid-points. We denote these oscillations spurious high frequencies. To illustrate this effect, we show in Fig. 7 the time evolution over a cardiac cycle of the ventricular pressure $P_{v}^{n}$, the aortic valve flux $K_{\mathrm{ar}}^{-1}\left|P_{v}^{n}-P_{\mathrm{ar}}^{n}\right|_{+}$and the proximal arterial pressure $P_{\mathrm{ar}}^{n}$ that are computed with the parameters chosen as in [47]. We compare three different configurations, $(\alpha, \theta)=(0,0.5)$ (no artificial viscosity), $(\alpha, \theta)=(0,0.75)$ (artificial viscosity only on $\left.P_{v}\right)$ and $(\alpha, \theta)=$ $(1,0.75)$ (artificial viscosity on $\dot{y}$ and $P_{v}$ ). It can be seen that the latter case avoids all kinds of spurious frequencies while preserving, qualitatively, the consistency of the approximation and the cost of the numerical scheme. Of note, the artificial viscosity decreases the formal order of accuracy of the scheme, an acceptable price to pay as our time-step is already rather small to account for the stiff parts of the pressure variation. 


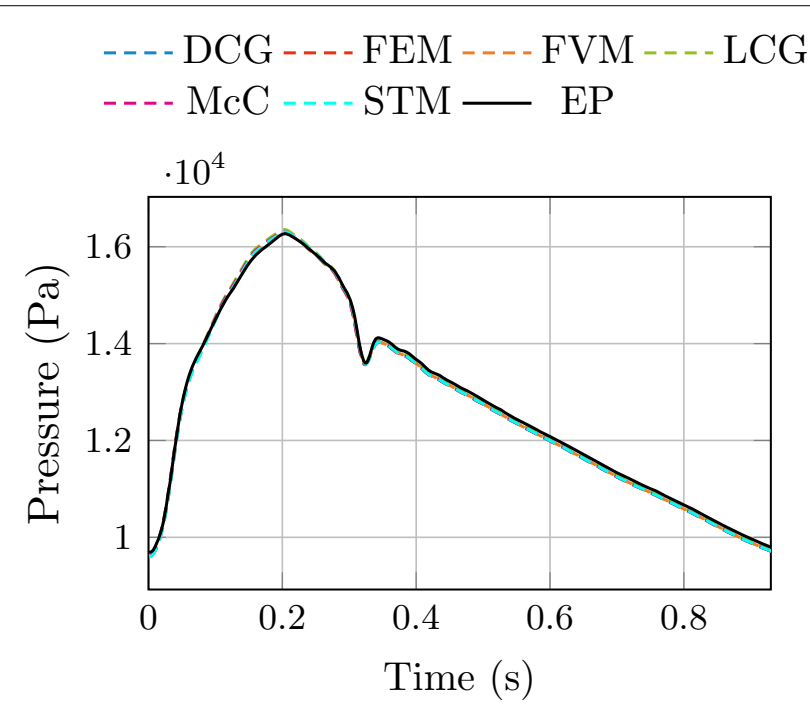

Fig. 6 Validation of the proposed energy-preserving formulation through the comparison of the results of our approach and those reported in [18]. DCG discontinuous Galerkin, FEM Galerkin least-squares finite element method, FVM finite volume method, LCG locally conservative Galerkin, McC finite difference MacCormack method, STM simplified trapezium rule method, EP energy-preserving scheme proposed in this work

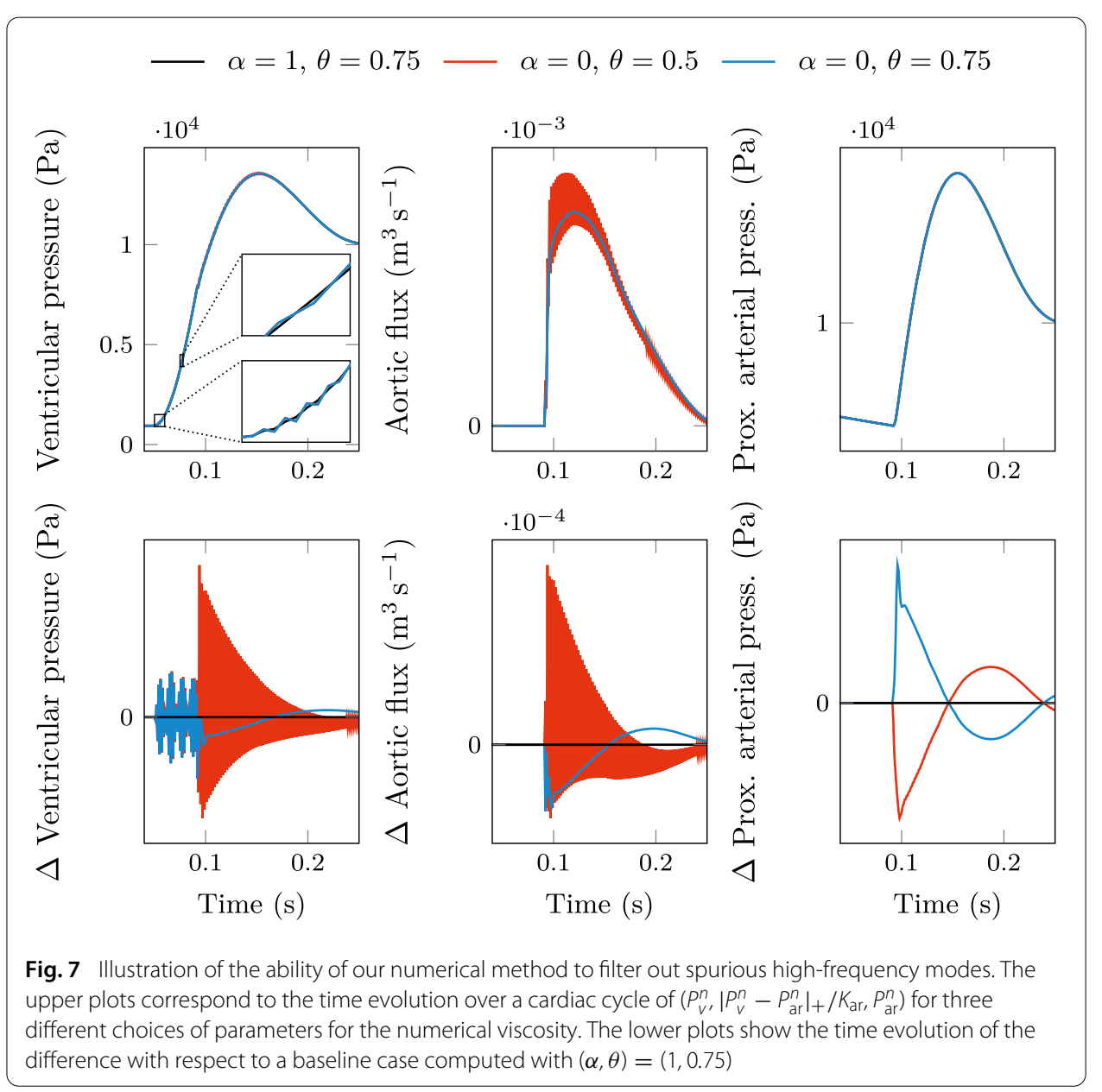



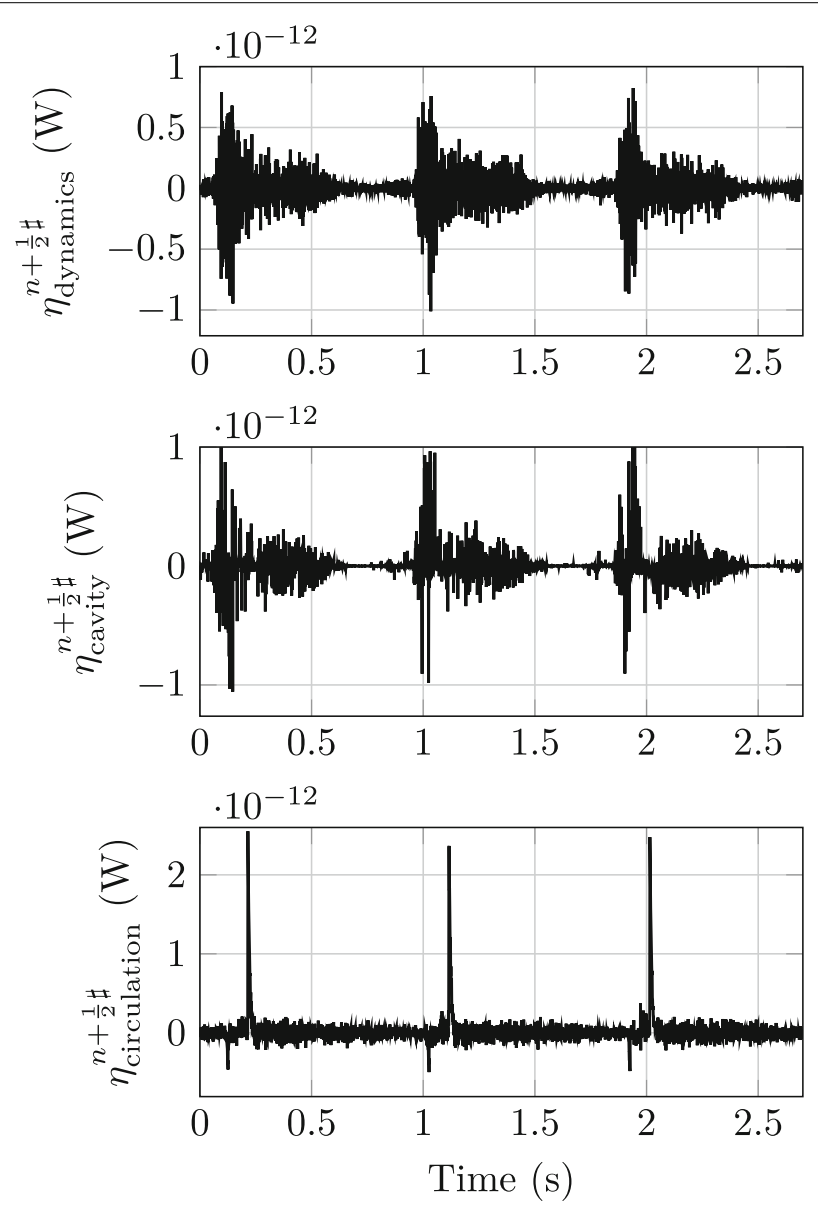

Fig. 8 Illustration that the energy balances are satisfied at the discrete level. The energy conservation residuals (66), (77) and (79) are evaluated numerically over time

\section{Numerical validation of the energy balance behind the coupling strategy}

As an illustration of the validity of the energy-preserving numerical methods developed in this work, we present the numerical evaluation of the energy conservation residuals of the three main model elements (66), (77) and (79). For that, we define the residuals as

$$
\left\{\begin{aligned}
\eta_{\text {dynamics }}^{n+\frac{1}{2} \sharp}= & \frac{\mathcal{E}_{\mathrm{hr}}^{n+1}-\mathcal{E}_{\mathrm{hr}}^{n}}{\Delta t_{n}}+\frac{\mathcal{E}_{\mathrm{hr}, \text { num }}^{n+1}-\mathcal{E}_{\mathrm{hr}, \text { num }}^{n}}{\Delta t_{n}}+\frac{\mathcal{E}_{c}^{n+1}-\mathcal{E}_{c}^{n}}{\Delta t_{n}} \\
& +\mathcal{D}_{h r}^{n+\frac{1}{2}}+\mathcal{D}_{\mathrm{hr}, \mathrm{num}}^{n+\frac{1}{2} \sharp}+\mathcal{D}_{c}^{n+\frac{1}{2}}-\dot{\mathcal{W}}^{n+\frac{1}{2} \sharp}-\mathcal{P}_{\mathrm{ATP}}^{n+\frac{1}{2} \sharp} \\
\eta_{\text {cavity }}^{n+\frac{1}{2} \sharp}= & \frac{\mathcal{E}_{v}^{n+1}-\mathcal{E}_{v}^{n}}{\Delta t_{n}}+\mathcal{D}_{v}^{n+\theta}+\mathcal{D}_{v, \text { num }}^{n+\frac{1}{2} \sharp}-\mathcal{P}_{\mathrm{ATP}}^{n+\frac{1}{2} \sharp}+\dot{\mathcal{W}}^{n+\frac{1}{2} \sharp}+P_{\mathrm{ar}}^{n+\frac{1}{2}} Q_{\mathrm{ar}}^{n+\frac{1}{2} \sharp}, \\
\eta_{\text {circulation }}^{n+\frac{1}{2} \sharp}= & \frac{\mathcal{E}_{\mathrm{ar}}^{n+1}-\mathcal{E}_{\mathrm{ar}}^{n}}{\Delta t_{n}}+\frac{\mathcal{E}_{w}^{n+1}-\mathcal{E}_{w}^{n}}{\Delta t_{n}}+\mathcal{D}_{\mathrm{ar}}^{n+\frac{1}{2}}+\mathcal{D}_{w}^{n+\frac{1}{2}}-P_{\mathrm{ar}}^{n+\frac{1}{2}} Q_{\mathrm{ar}}^{n+\frac{1}{2} \sharp} .
\end{aligned}\right.
$$

Their time evolution is presented in Fig. 8 over several heart cycles for the simulation with the parameters inspired from [47] and whose results are presented in Figs. 9 and 10. We can notice that the magnitude of the residuals is 12 orders of magnitude lower than that of the individual energy fluxes that compose them. This result thus validates the fact that the energy balances are indeed satisfied at the discrete level. 

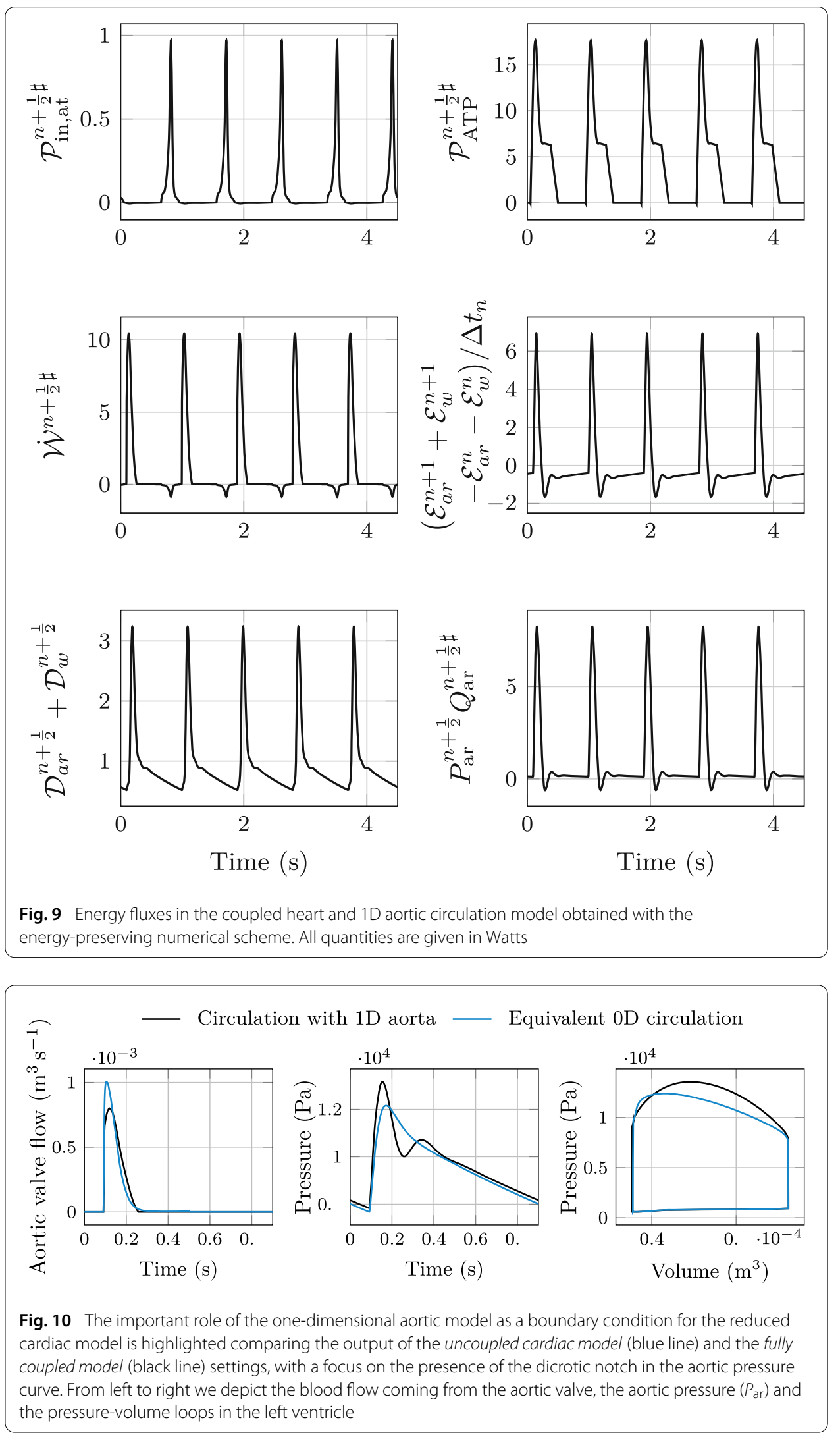
Having an energy-preserving formulation for the discretization of the model equations ensures that we can make sense of the individual terms of the energy balance. On the one hand, it allows to get a better picture on the solution of the model equations by analyzing the fluxes of energy between the different elements of the model. We present in Fig. 9 some of the energy fluxes that can be computed. We define the following notations

$$
\left\{\begin{array}{l}
\mathcal{P}_{\mathrm{in}, \mathrm{at}}^{n+\frac{1}{2} \sharp}=\frac{||_{\mathrm{at}}^{n+\frac{1}{2}}-P_{v}^{n+\theta} \mid+}{K_{\mathrm{at}}} P_{\mathrm{at}}^{n+\frac{1}{2}}-\frac{\left|P_{v}^{n+\theta}-P_{\mathrm{at}}^{n+\frac{1}{2}}\right|+}{K_{a r}} P_{\mathrm{at}}^{n+\frac{1}{2}}, \\
\dot{\mathcal{W}}^{n+\frac{1}{2} \sharp}=-P_{v}^{n+\theta} \frac{V\left(y^{n+1}\right)-V\left(y^{n}\right)}{\Delta t_{n}}, \\
\mathcal{P}_{\mathrm{ATP}}^{n+\frac{1}{2} \sharp}=\frac{\left|\Omega_{0}\right| n_{0}\left(e_{c}^{n}\right)}{\sqrt{k_{c}^{n+1}}}\left(\sigma_{0} \lambda_{c}^{n+\frac{1}{2}}-\frac{k_{0}\left(\lambda_{c}^{n+\frac{1}{2}}\right)^{2}}{2 \sqrt{k_{c}^{n+1}}}\right)\left|v^{n+1}\right|_{+} .
\end{array}\right.
$$

In the numerical results, we see how the contraction of the atrium creates an initial flux of energy $\mathcal{P}_{\text {in,at }}$ towards the left ventricle. Then, the chemical energy flux $\mathcal{P}_{\text {ATP }}$ provided at the micro level is transformed into the mechanical power $\dot{\mathcal{W}}$ by the left ventricle. It can be noted that the model predicts an energetic yield $\int_{t}^{t+T}|\mathcal{W}(\tau)|_{+} \mathrm{d} \tau / \int_{t}^{t+T} \mathcal{P}_{\mathrm{ATP}}(\tau) \mathrm{d} \tau$ of $26.2 \%$, which lies in the physiological range: 25 to $35 \%[48,49]$. The average power produced by the ventricle over a heartbeat is $1.02 \mathrm{~W}$, which is in accordance with the values obtained experimentally $[47,50]$. Moreover, the peak of mechanical power reaches $10.4 \mathrm{~W}$, which is consistent with the evaluation of this quantity from other mechanical models for patients suffering from aortic valve disease [51]. The mechanical power is then transferred with little dissipation through the valves into the aorta that receives the influx of energy $P_{\mathrm{ar}} Q_{\mathrm{ar}}$. On the other hand, being able to compute the individual energy fluxes inside the model is of great interest from an application point of view, since some of the terms of the energy balance are indeed used by medical doctors. For instance, anesthetists are interested in the work developed by the left ventricle for the monitoring of the heart [47] during surgical intervention, wheres cardiologists see this same parameter as a potential biomarker for the evaluation of left ventricle dysfunctions [50] and the myocardial efficiency is considered as a relevant indicator to assess the state of patients having aortic or valve pathologies $[52,53]$.

\section{Physiological outcomes of the coupling}

In this section, we examine the physiological interest of coupling the arterial and the cardiac model. The three model settings described above are involved to highlight the differences in the results when one of the two components, i.e. the cardiac model or the one-dimensional vessel, is not taken into account-the fully coupled model allowing to study the effect of the mutual interplay between the heart and the arterial network.

\section{Importance of the downstream circulation for the heart: the dicrotic notch}

The physiological arterial pressure curve shows two main parts: the systolic and the diastolic phase. During the first phase, the heart ejects the blood into the aortic root and the arterial pressure increases rapidly and reaches a peak, known as the systolic pressure value. Then, the pressure starts falling but it is interrupted by an incisura, known as the dicrotic notch, that happens at the time of the passage to the diastolic phase and causes a second peak. The pressure then continues its downslope to its minimum, the diastolic pressure $[54,55]$. Any change in the shape of this curve represents a modification in the vessel 
condition, that is why it is important to be able to properly reproduce these features. We focus here on the reproduction of the dicrotic notch. For that purpose, we perform a simulation using the uncoupled cardiac model and the fully coupled model and we compare the results.

From the results presented in Fig. 10 we can observe that only the pressure curve obtained with the fully coupled model (black curve in the center) shows a dicrotic notch. Moreover, this was obtained with an inflow condition (in black in the left box), coming from the heart, which does not present a backflow. This confirms the latest findings of [21] and suggests that the dicrotic notch is not caused by the presence of the backflow but can be observed when wave propagation phenomena are represented. In addition, although both settings are able to reproduce a physiological shape for the curves, there are more differences between the two results beyond the reproducibility of the dicrotic notch. First, we can observe that the peak of the blood flow is slightly increased when the lumped-parameter model is used to represent the full circulation. Moreover, from the pressure-volume loops (in the right box) we can notice a distinction in shape between the two ejections. This suggests that the systolic blood pressure reaches a lower peak value when the circulation is completely represented by a lumped-parameter model. The last consideration is confirmed even more clearly in the pressure curves, since the lumpedparameter model is able to fit the diastolic decay of pressure but it is not sufficient to properly reproduce the systolic peak [46] and, more generally, the systolic phase. In fact, only when a distributed aortic model is considered it is possible to obtain a more physiological wave showing a noticeable dicrotic notch. These results suggest that when a cardiovascular model is used to analyze phenomena strongly associated with the ventriculo-arterial interaction, lumped-parameter models are not sufficient to represent the downstream circulation and it is necessary to employ a higher-dimensional model (e.g. a 1D-model) that is able to capture the influence of pulse wave transmission within the circulation.

\section{Importance of the heart for the aorta: ageing}

During ageing, the vessel walls undergo a degeneration of elastin fibers, a decrease in smooth muscle and an increase in collagen. These changes cause the stiffening of the arteries and in particular of the aorta. As a result, it is observed that the systolic peak pressure increases and the aortic and left ventricular late systolic pressure augment, whereas the aortic blood flow peak and the diastolic pressure decrease. These features are indeed commonly observed among elderly subjects. Of note, the effects of ageing on the heart are minimal with respect to those in the main arteries [56].

As it was done in [29], we use the simulation of ageing to highlight the importance of accounting for heart-circulation interactions in cardiovascular modeling. In order to simulate the ageing of the vessel, we modify some relevant arterial parameters. We follow here the scheme proposed by [57], carrying out the appropriate manipulations to adapt this strategy to our model (single one-dimensional vessel). The parameter $\beta$, which is a surrogate of the arterial wall stiffness, the total arterial resistance and the total compliance are modified to represent "older" vessels. To take into account the contribution of the 1D model to the total resistance, the average pressure observed at the beginning of the aorta $\left(\bar{P}_{\text {inlet }}\right)$ is used as a marker for the impedance of the vessel. A target value $\left(\bar{P}_{\text {target }}\right)$ for this inlet pressure is calculated in order to induce the increasing pressure that is observed in 


\section{Uncoupled aortic model with imposed inlet flow}
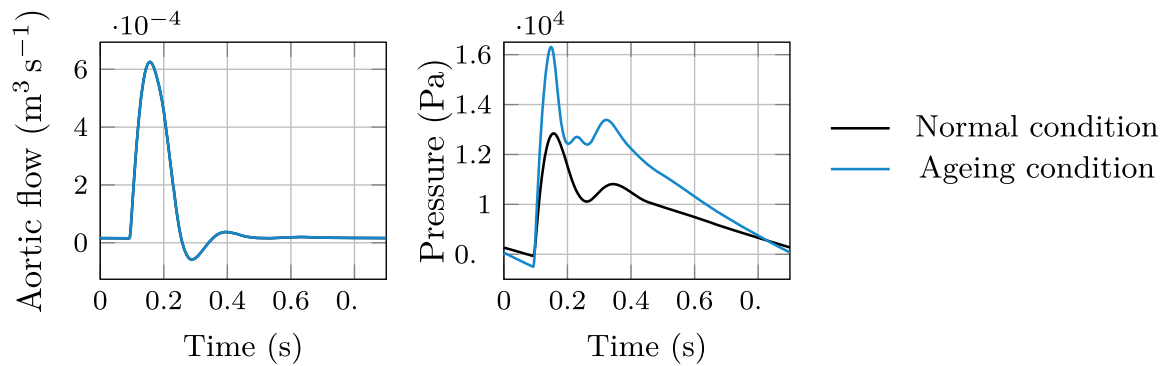

Fully coupled heart-circulation model
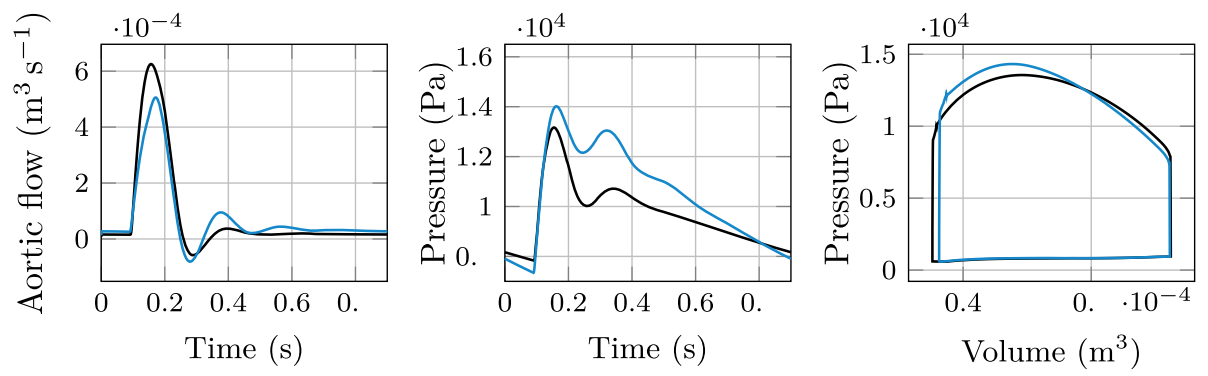

Fig. 11 The important role of the cardiac model as an inlet condition for the distributed arterial model is highlighted comparing the output of the uncoupled aortic model (top left and right) and the fully coupled model (bottom left to right) settings, with a focus on the effects of ageing on the elastic arteries. On the left we depict the blood flow at the first element of the aortic model, in the center we show the aortic pressure $\left(P_{\text {ar }}\right)$ at the same space element and at the bottom right we depict the pressure-volume loops in the left ventricle, for normal physiological conditions (black line) and for an arterial ageing conditions (blue line)

normal ageing, and it is chosen as a $10 \%$ increase from the baseline inlet pressure. The peripheral resistance $-R_{\text {per }}$ in our RCR model - is updated conforming to

$$
R_{\text {per }, k+1}+R_{c}=\frac{\bar{P}_{\text {target }}}{\bar{P}_{\text {inlet }, k}}\left(R_{\text {per }, k}+R_{c}\right),
$$

where $k$ is the ageing process iterative index. The parameter $\beta$ is then modified as $\beta_{\text {new }}=2.5 \beta$. The total arterial compliance is decreased as $C_{\text {tot,new }}=C_{\text {tot }} / 2$, with the total compliance $C_{\text {tot }}$ being defined as

$$
C_{\mathrm{tot}}=C_{1 \mathrm{D}}+C_{0 \mathrm{D}, \text { distal }}+C_{0 \mathrm{D}, \text { transmission }}
$$

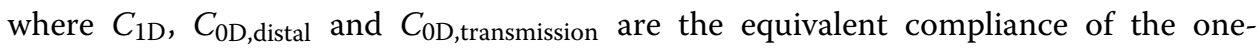
dimensional arterial model, the distal Windkessel model and the transmission model (if present), respectively. Then, $C_{1 \mathrm{D}, \text { new }}$ is updated according to the changes in $\beta-$ see (85)and $C_{0 \mathrm{D} \text {,distal,new }}$ is obtained from

$$
C_{0 \mathrm{D} \text {,distal,new }}=C_{\mathrm{tot}, \text { new }}-C_{1 \mathrm{D} \text {,new }}-C_{0 \mathrm{D} \text {,transmission }} .
$$

The simulation of ageing is performed with the uncoupled aortic model and the fully coupled model settings.

The obtained results are presented in Fig. 11. In both simulation settings, we can observe numerous changes with respect to the baseline case that are typical of the ageing process: higher systolic peak, lower diastolic pressure and a small increase in wave propagation speed (the dicrotic notch is anticipated). However, the pressure curve obtained with the 
coupled model is more physiological. In particular, it is possible to see a trend towards the merging of the pressure systolic peak and the dicrotic peak, as it happens when the ageing process is advanced [56], and the dicrotic notch fades away. Indeed, without a cardiac model the inflow has to be imposed and therefore it does not adapt to the arterial conditions. In the ageing case with the uncoupled aortic model it is possible to observe a double reflection that does not reflect the arterial response to ageing. Furthermore, when using a coupled model, the blood flow is impacted itself by the ageing process. Our simulation shows a decreased peak compared to the baseline case. Finally, the use of a coupled heartcirculation model allows to compute the variation of the left ventricular pressure-volume loop as a result of the ageing process. This enables to monitor the quantitative effects of the arterial ageing on the cardiac function.

\section{Conclusions}

In this work, we present an original method to couple reduced-order blood flow circulation models and heart models through the point of view of energy balance both at the continuous and the discrete level. This is a difficult path as model reduction is often associated with the loss of energy balances for limit models. In fact, our coupled formulation is here proven to satisfy a full energy balance at the continuous level, with an additional consistent and controlled numerical dissipation on the fully discrete scheme. This allows to control the energy sources avoiding instabilities such as for instance uncontrolled back flows or perpetual motion cardiac engines. Essentially, our controlled coupling improves the modeling from the cardiac side and from the cardiovascular side. On the one hand, from the cardiac point of view we obtain better physiological signals with respect to the completely lumped heart-plus-Windkessel model proposed in [32]. On the other hand, we introduce a more physiological heart engine than a phenomenological time-varying elastance model acting as a flow generator in [29]. We believe that this paves the way for suitably investigating physiological aspects of heart-circulation coupling, as illustrated here with the dicrotic notch and the ageing process.

\section{Authors' contributions}

JM, FC and SI contributed to the conception, analysis, implementation, interpretation of the reduced arterial model. JM, PM, FK contributed to conception, analysis, implementation, interpretation of the heart-arterial coupling. All authors revised the methods and contributed to the drafting and revising of the manuscript.

Funding

Authors knowledge the support of the CNRS GdR 3587 AMORE.

\section{Declarations}

Competing interests

The authors declare that they have no competing interests.

Author details

'Inria, Team MEDISIM, Inria Saclay-lle-de-France, 91128 Palaiseau, France, ${ }^{2}$ LMS, Ecole Polytechnique, CNRS, Institut Polytechnique de Paris, 91128 Palaiseau, France, ${ }^{3}$ Institute of Mathematics and Scientific Computing, NAWI Graz, University of Graz, Graz, Austria.

\section{Appendix: Details on the construction of the approximate radius function}

In this appendix we describe how the discrete function $r_{h}(\Phi)$ is constructed. This construction is based on a pre-computation step in which the approximate tube law $r_{h}(\Phi)$ is sought as a bijective $C^{1}$-functions of $\mathbb{R}$ to $\mathbb{R}^{+}$that corresponds to a quadratic piecewise 
approximation of $r(\Phi)$ on an interval $\left[\Phi_{0}, \Phi_{N}\right]$ and some smooth analytic functions over $\left(-\infty, \Phi_{0}\right]$ and $\left[\Phi_{N},+\infty\right)$. After this pre-computations step the values of $r_{h}(\Phi)$ and $r_{h}^{\prime}(\Phi)$ can be obtained either by quadratic (or linear) interpolation or evaluation of one (or two) transcendental functions. We first choose a set of points

$$
\left\{\Phi_{n}\right\}_{n=0}^{n=N} \text { with } \quad \Phi_{n}<\Phi_{n+1}
$$

that represents a sufficiently fine sampling of the interval $\left[\Phi_{0}, \Phi_{N}\right]$. Then we compute $r\left(\Phi_{0}\right)$ and $r^{\prime}\left(\Phi_{n}\right)$ for each $n \in\{0, \ldots, N\}$, using Eqs. (35) and (36) and the relation $r(\Phi)=$ $\varphi^{-1}(\Phi)$. For each $n \in\{0, \ldots, N\}$ the value $r^{\prime}\left(\Phi_{n}\right)$ corresponds to the value of the function $r_{h}^{\prime}\left(\Phi_{n}\right)$ and for $\Phi \in\left[\Phi_{n-1}, \Phi_{n}\right]$ the value of $r_{h}^{\prime}(\Phi)$ is obtained by affine interpolation. More precisely

$$
r_{h}^{\prime}(\Phi)=\frac{\Phi-\Phi_{n-1}}{\Phi_{n}-\Phi_{n-1}} r_{h}^{\prime}\left(\Phi_{n}\right)+\frac{\Phi_{n}-\Phi}{\Phi_{n}-\Phi_{n-1}} r_{h}^{\prime}\left(\Phi_{n-1}\right), \quad \Phi \in\left[\Phi_{n-1}, \Phi_{n}\right] .
$$

The function $r_{h}(\Phi)$ for $\Phi \in\left[\Phi_{0}, \Phi_{N}\right]$ is obtained by integrating the function $r_{h}^{\prime}(\Phi)$ between $\Phi_{0}$ and $\Phi$ and setting $r_{h}\left(\Phi_{0}\right)=r\left(\Phi_{0}\right)$. In practice the values $\left\{r_{h}\left(\Phi_{n}\right)\right\}_{n=0}^{n=N}$ are tabulated. They are given by for $n \in\{1, \ldots, N\}$

$$
r_{h}\left(\Phi_{0}\right)=r\left(\Phi_{0}\right) \quad \text { and } \quad r_{h}\left(\Phi_{n}\right)=r_{h}\left(\Phi_{n-1}\right)+\left(\Phi_{n}-\Phi_{n-1}\right) \frac{r^{\prime}\left(\Phi_{n}\right)+r^{\prime}\left(\Phi_{n-1}\right)}{2}
$$

and the function $r_{h}(\Phi)$ for $\Phi \in\left[\Phi_{n-1}, \Phi_{n}\right]$ is given by

$$
r_{h}(\Phi)=r_{h}\left(\Phi_{n-1}\right)+\frac{\frac{1}{2}\left(\Phi-\Phi_{n-1}\right)^{2}}{\Phi_{n}-\Phi_{n-1}} r_{h}^{\prime}\left(\Phi_{n}\right)+\frac{\frac{1}{2}\left(\Phi_{n}-\Phi_{n-1}\right)^{2}-\frac{1}{2}\left(\Phi_{n}-\Phi\right)^{2}}{\Phi_{n}-\Phi_{n-1}} r_{h}^{\prime}\left(\Phi_{n-1}\right) .
$$

For small values of $\Phi$ we prescribe an exponential behavior (following the modification of the model introduced in "Extension of the model in a non-physiological range" section),

$$
r_{h}(\Phi)=r\left(\Phi_{0}\right) e^{\frac{r^{\prime}\left(\Phi_{0}\right)}{r\left(\Phi_{0}\right)}\left(\Phi-\Phi_{0}\right)}, \quad \Phi \in\left(-\infty, \Phi_{0}\right] .
$$

A large value of $\Phi$ corresponds to a large value of $R$ in (35). For these values one can expect that $r(\Phi)$ behaves as an affine function of $\Phi^{\frac{2}{3}}$. Thus we set

$$
r_{h}(\Phi)=\frac{3}{2} \Phi_{N}^{\frac{1}{3}} r^{\prime}\left(\Phi_{N}\right) \Phi^{\frac{2}{3}}+r_{h}\left(\Phi_{N}\right)-\frac{3}{2} \Phi_{N} r^{\prime}\left(\Phi_{N}\right), \quad \Phi \in\left[\Phi_{N},+\infty\right) .
$$

Received: 7 January 2021 Accepted: 22 August 2021

Published online: 28 September 2021

\section{References}

1. Formaggia L, Nobile F, Quarteroni A, Veneziani A. Multiscale modelling of the circulatory system: a preliminary analysis. Comput Vis Sci. 1999;2(2-3):75-83. https://doi.org/10.1007/s007910050030.

2. Quarteroni A, Formaggia L. Mathematical modelling and numerical simulation of the cardiovascular system. In: Handbook of numerical analysis. Hoboken: Elsevier; 2004. p. 3-127. https://doi.org/10.1016/s1570-8659(03)12001-7.

3. Arts T, Delhaas T, Bovendeerd P, Verbeek X, Prinzen FW. Adaptation to mechanical load determines shape and properties of heart and circulation: the CircAdapt model. Am J Physiol Heart Circ Physiol. 2005:288(4):H1943-54.

4. Shi Y, Lawford P, Hose R. Review of Zero-D and 1-D models of blood flow in the cardiovascular system. BioMed Eng OnLine. 2011;10(1):33. https://doi.org/10.1186/1475-925x-10-33.

5. Manzoni A, Bonomi D, Quarteroni A. Reduced order modeling for cardiac electrophysiology and mechanics: new methodologies, challenges and perspectives. In: Mathematical and numerical modeling of the cardiovascular system and applications. Berlin: Springer; 2018. p. 115-66.

6. Westerhof N, Lankhaar JW, Westerhof BE. The arterial windkessel. Med Biol Eng Comput. 2009;47(2):131-41.

7. Segers $P$, Stergiopulos N, Westerhof N, Wouters $P$, Kolh P, Verdonck P. Systemic and pulmonary hemodynamics assessed with a lumped-parameter heart-arterial interaction model. J Eng Math. 2003;47(3-4):185-99.

8. Segers P, Rietzschel E, De Buyzere M, Stergiopulos N, Westerhof N, Van Bortel L, et al. Three-and four-element Windkessel models: assessment of their fitting performance in a large cohort of healthy middle-aged individuals. Proc Inst Mech Eng H. 2008;222(4):417-28.

9. Quarteroni A, Veneziani A, Vergara C. Geometric multiscale modeling of the cardiovascular system, between theory and practice. Comput Methods Appl Mech Eng. 2016;302:193-252. https://doi.org/10.1016/j.cma.2016.01.007. 
10. Sainte-Marie J, Chapelle D, Cimrman R, Sorine M. Modeling and estimation of the cardiac electromechanical activity. Comput Struct. 2006;84(28):1743-59.

11. Kerckhoffs RC, Neal ML, Gu Q, Bassingthwaighte JB, Omens JH, McCulloch AD. Coupling of a 3D finite element model of cardiac ventricular mechanics to lumped systems models of the systemic and pulmonic circulation. Ann Biomed Eng. 2007;35(1):1-18.

12. Augustin CM, Neic A, Liebmann M, Prassl AJ, Niederer SA, Haase G, et al. Anatomically accurate high resolution modeling of human whole heart electromechanics: a strongly scalable algebraic multigrid solver method for nonlinear deformation. J Comput Phys. 2016;305:622-46.

13. Hirschvogel M, Bassilious M, Jagschies L, Wildhirt SM, Gee MW. A monolithic 3D-OD coupled closed-loop model of the heart and the vascular system: experiment-based parameter estimation for patient-specific cardiac mechanics. Int J Numer Methods Biomed Eng. 2017;33(8):e2842.

14. Alastruey J, Parker KH, Sherwin SJ. Lumped parameter outflow models for 1-D blood flow simulations: effect on pulse waves and parameter estimation. Commun Comput Phys. 2008;4:317-36.

15. Vignon-Clementel I, Arbia G, Baretta A, Corsini C, Esmaily Moghadam M, Troianowski G, et al. From patient-specific data to multiscale hemodynamics simulations: the challenge of boundary conditions. In: Middleton J, editor. 10th international symposium on computer methods in biomechanics and biomedical engineering. ISBN: 978-0-95621215-3. Berlin, Germany: Arup; 2012. p. 603-8. https://hal.inria.fr/hal-00765819.

16. Guan D, Liang F, Gremaud PA. Comparison of the Windkessel model and structured-tree model applied to prescribe outflow boundary conditions for a one-dimensional arterial tree model. J Biomech. 2016;49(9):1583-92. https://doi. org/10.1016/j.jbiomech.2016.03.037.

17. Matthys KS, Alastruey J, Peiró J, Khir AW, Segers P, Verdonck PR, et al. Pulse wave propagation in a model human arteria network: assessment of 1-D numerical simulations against in vitro measurements. J Biomech. 2007;40(15):3476-86 https://doi.org/10.1016/j.jbiomech.2007.05.027.

18. Boileau E, Nithiarasu P, Blanco PJ, Müller LO, Fossan FE, Hellevik LR, et al. A benchmark study of numerical schemes for one-dimensional arterial blood flow modelling. Int J Numer Methods Biomed Eng. 2015;31(10):e02732. https:// doi.org/10.1002/cnm.2732.

19. Reymond P, Merenda F, Perren F, Rüfenacht D, Stergiopulos N. Validation of a one-dimensional model of the systemic arterial tree. Am J Physiol Heart Circ Physiol. 2009;297(1):H208-22. https://doi.org/10.1152/ajpheart.00037.2009.

20. Bollache E, Kachenoura N, Redheuil A, Frouin F, Mousseaux E, Recho P, et al. Descending aorta subject-specific onedimensional model validated against in vivo data. J Biomech. 2014;47(2):424-31. https://doi.org/10.1016/j.jbiomech. 2013.11.009.

21. Politi MT, Ghigo A, Fernández JM, Khelifa I, Gaudric J, Fullana JM, et al. The dicrotic notch analyzed by a numerical model. Comput Biol Med. 2016;72:54-64. https://doi.org/10.1016/j.compbiomed.2016.03.005.

22. Pontrelli G. A multiscale approach for modelling wave propagation in an arterial segment. Comput Methods Biomech Biomed Eng. 2004;7(2):79-89. https://doi.org/10.1080/1025584042000205868.

23. Xiao N, Alastruey J, Figueroa CA. A systematic comparison between 1-D and 3-D hemodynamics in compliant arterial models. Int J Numer Methods Biomed Eng. 2013:30(2):204-31. https://doi.org/10.1002/cnm.2598.

24. Willemet M, Alastruey J. Arterial pressure and flow wave analysis using time-domain 1-D hemodynamics. Ann Biomed Eng. 2014;43(1):190-206. https://doi.org/10.1007/s10439-014-1087-4

25. Müller LO, Toro EF. A global multiscale model for the human circulation with emphasis on the venous system. Int $J$ Numer Methods Biomed Eng. 2014;Published online in Wiley Online Library (wileyonlinelibrary.com). https://doi.org/ 10.1002/cnm.2622.Preprint available at http://www.newton.ac.uk/preprints/NI13007.pdf.

26. Mynard JP, Smolich JJ. One-dimensional haemodynamic modeling and wave dynamics in the entire adult circulation. Ann Biomed Eng. 2015. https://doi.org/10.1007/s10439-015-1313-8.

27. Brault A, Dumas $L$, Lucor D. Uncertainty quantification of inflow boundary condition and proximal arterial stiffness coupled effect on pulse wave propagation in a vascular network. Int J Numer Methods Biomed Eng. 2017;33(10):e2859.

28. Audebert C, Bucur P, Bekheit M, Vibert E, Vignon-Clementel I, Gerbeau JF. Kinetic scheme for arterial and venous blood flow, and application to partial hepatectomy modeling. Comput Methods Appl Mech Eng. 2017:314:102-25.

29. Formaggia L, Lamponi D, Tuveri M, Veneziani A. Numerical modeling of $1 D$ arterial networks coupled with a lumped parameters description of the heart. Comput Methods Biomecha Biomed Eng. 2006;9(5):273-88. https://doi.org/10. 1080/10255840600857767.

30. Shishido T, Hayashi K, Shigemi K, Sato T, Sugimachi M, Sunagawa K. Single-beat estimation of end-systolic elastance using bilinearly approximated time-varying elastance curve. Circulation. 2000;102(16):1983-9.

31. Lumens J, Delhaas T, Kirn B, Arts T. Three-wall segment (TriSeg) model describing mechanics and hemodynamics of ventricular interaction. Ann Biomed Eng. 2009;37(11):2234-55.

32. Caruel M, Chabiniok R, Moireau P, Lecarpentier Y, Chapelle D. Dimensional reductions of a cardiac model for effective validation and calibration. Biomech Model Mechanobiol. 2014:13(4):897-914.

33. Lombardi D. Inverse problems in 1D hemodynamics on systemic networks: a sequential approach. Int J Numer Methods Biomed Eng. 2013;30(2):160-79.

34. Caiazzo A, Caforio F, Montecinos G, Müller LO, Blanco PJ, Toro EF. Assessment of reduced-order unscented Kalman filter for parameter identification in 1-dimensional blood flow models using experimental data. Int J Numer Methods Biomed Eng. 2017:33(8):e2843.

35. Arthurs CJ, Xiao N, Moireau P, Schaeffter T, Figueroa CA. A flexible framework for sequential estimation of model parameters in computational hemodynamics. Adv Model Simul Eng Sci. 2020;7:1-37.

36. Chapelle D, Le Tallec P, Moireau P, Sorine M. An energy-preserving muscle tissue model: formulation and compatible discretizations. Int J Multiscale Comput Eng. 2012;10(2):189-211.

37. Formaggia L, Gerbeau JF, Nobile F, Quarteroni A. On the coupling of 3D and 1D Navier-Stokes equations for flow problems in compliant vessels. Comput Methods Appl Mech Eng. 2000;191(561_-582):6-7.

38. Westerhof N, Bosman F, De Vries CJ, Noordergraaf A. Analog studies of the human systemic arterial tree. J Biomech. 1969:2(2):121-43. 
39. Burtschell B. Mechanical modeling and numerical methods for poromechanics : application to myocardium perfusion 2016. 2016SACLX022. http://www.theses.fr/2016SACLX022/document.

40. Kimmig F, Chapelle D, Moireau P. Thermodynamic properties of muscle contraction models and associated discretetime principles. Adv Model Simul Eng Sci. 2019;6(1):6. https://doi.org/10.1186/s40323-019-0128-9.

41. Holzapfel GA, Ogden RW. Constitutive modelling of passive myocardium: a structurally based framework for material characterization. Phil Trans R Soc A. 2009:367:3445-75.

42. Bestel J, Clément F, Sorine M. A biomechanical model of muscle contraction. Med Image Comput Comput Assisted Intervent MICCAI 2001. 2001;2208(2):1159-61.

43. Chabiniok R, Moireau P, Kiesewetter C, Hussain T, Razavi R, Chapelle D. Assessment of atrioventricular valve regurgitation using biomechanical cardiac modeling. In: Imaging and modelling of the heart. New York: International Publishing; 2017. p. 401-11. https://doi.org/10.1007/978-3-319-59448-4_38.

44. Hauret $P$, Le Tallec $P$. Energy-controlling time integration methods for nonlinear elastodynamics and low-velocity impact. Comput Methods Appl Mech Eng. 2006;195(37-40):4890-916.

45. Gonzalez O. Exact energy and momentum conserving algorithms for general models in nonlinear elasticity. Comput Methods Appl Mech Eng. 2000;190(13-14):1763-83.

46. Epstein S, Willemet M, Chowienczyk PJ, Alastruey J. Reducing the number of parameters in 1D arterial blood flow modeling: less is more for patient-specific simulations. Am J Physiol Heart Circu Physiol. 2015;309(1):H222-34. https:// doi.org/10.1152/ajpheart.00857.2014.

47. Le Gall A, Vallee F, Pushparajah K, Hussain T, Mebazaa A, Chapelle D, et al. Monitoring of cardiovascular physiology augmented by a patient-specific biomechanical model during general anesthesia. A proof of concept study. PLoS ONE. 2020;15(5):e0232830.

48. Schipke JD. Cardiac efficiency. Basic Res Cardiol. 1994;89(3):207-40

49. Knaapen P, Germans T, Knuuti J, Paulus WJ, Dijkmans PA, Allaart CP, et al. Myocardial energetics and efficiency: current status of the noninvasive approach. Circulation. 2007:115(7):918-27.

50. Dini FL, Guarini G, Ballo P, Carluccio E, Maiello M, Capozza P, et al. The left ventricle as a mechanical engine: from Leonardo da Vinci to the echocardiographic assessment of peak power output-to-left ventricular mass. J Cardiovasc Med. 2013;14(3):214-20

51. Gsell MA, Augustin CM, Prassl AJ, Karabelas E, Fernandes JF, Kelm M, et al. Assessment of wall stresses and mechanical heart power in the left ventricle: finite element modeling versus Laplace analysis. Int J Numer Methods Biomed Eng. 2018;34(12):e3147.

52. Güçlü A, Knaapen $P$, Harms HJ, Vonk AB, Stooker W, Groepenhoff $H$, et al. Myocardial efficiency is an important determinant of functional improvement after aortic valve replacement in aortic valve stenosis patients: a combined PET and CMR study. Eur Heart J Cardiovasc Imaging. 2015;16(8):882-9.

53. Fernandes JF, Goubergrits L, Brüning J, Hellmeier F, Nordmeyer S, da Silva TF, et al. Beyond pressure gradients: the effects of intervention on heart power in aortic coarctation. PLoS ONE. 2017;12(1):e0168487.

54. Mackanzie J. Study of the pulse, arterial, venous and hepatic and of the movements of the heart. JAMA J Am Med Assoc. 1902;XXXIX(11):648. https://doi.org/10.1001/jama.1902.02480370056022.

55. Esper SA, Pinsky MR. Arterial waveform analysis. Best Pract Res Clin Anaesthesiol. 2014;28(4):363-80. https://doi.org/ 10.1016/j.bpa.2014.08.002.

56. Vlachopoulos C, O'Rourke M, Nichols WW. McDonald's blood flow in arteries. Boca Raton: CRC Press; 2011. https:// doi.org/10.1201/b13568.

57. Fossan FE, Mariscal-Harana J, Alastruey J, Hellevik LR. Optimization of topological complexity for one-dimensional arterial blood flow models. J R Soc Interface. 2018;15(149):20180546. https://doi.org/10.1098/rsif.2018.0546.

\section{Publisher's Note}

Springer Nature remains neutral with regard to jurisdictional claims in published maps and institutional affiliations.

\section{Submit your manuscript to a SpringerOpen ${ }^{\circ}$ journal and benefit from:}

- Convenient online submission

- Rigorous peer review

- Open access: articles freely available online

- High visibility within the field

Retaining the copyright to your article

Submit your next manuscript at $\mathbf{s p r i n g e r o p e n . c o m ~}$ 